\title{
Some implications between Grothendieck's anabelian conjectures
}

\author{
Giulio Bresciani
}

\begin{abstract}
Grothendieck gave two forms of his "main conjecture of anabelian geometry", namely the section conjecture and the hom conjecture. He stated that these two forms are equivalent and that if they hold for hyperbolic curves, then they hold for elementary anabelian varieties too. We state a stronger form of Grothendieck's conjecture (equivalent in the case of curves) and prove that Grothendieck's statements hold for our form of the conjecture. We work with DM stacks, rather than schemes. If $X$ is a DM stack over $k \subseteq \mathbb{C}$, we prove that whether $X$ satisfies the conjecture or not depends only on $X_{\mathbb{C}}$. We prove that the section conjecture for hyperbolic orbicurves stated by Borne and Emsalem follows from the conjecture for hyperbolic curves.
\end{abstract}

\section{Introduction}

\subsection{The main conjecture of anabelian geometry}

In his letter to Faltings [Gro97], Grothendieck gave two forms of his "main conjecture of anabelian geometry", the hom conjecture and the section conjecture, for anabelian varieties. He refrained from defining precisely the class of anabelian varieties, but he said that it certainly contained smooth, hyperbolic curves and the so-called elementary anabelian varieties, that is, those obtained by subsequent fibrations from smooth hyperbolic curves. He said that being anabelian is a purely geometric property, namely whether $X / k$ is anabelian depends only on $X_{\bar{k}}$, or $X_{\mathbb{C}}$ if $k \subseteq \mathbb{C}$, and that if the main conjecture holds for proper, hyperbolic curves, then it holds for proper, elementary anabelian varieties.

Let us recall the two forms of the main conjecture. If $G$ and $H$ are extensions of a group $\Gamma$, then Hom-ext ${ }_{\Gamma}(G, H)$ is the set of homomorphisms $G \rightarrow H$ which commute with the projection to $\Gamma$ modulo the natural action of $\operatorname{ker}(H \rightarrow \Gamma)$ by conjugation. If $k$ is a field, we denote by $\Gamma_{k}=\operatorname{Gal}\left(k^{s} / k\right)$ the absolute Galois group. If $T$ and $X$ are geometrically connected over $k$ with étale fundamental groups $\pi_{1}(T)$ and $\pi_{1}(X)$ (we omit base points), there is a natural map

$$
\operatorname{Hom}_{k}(T, X) \rightarrow \operatorname{Hom}_{-\operatorname{ext}_{\Gamma_{k}}}\left(\pi_{1}(T), \pi_{1}(X)\right) .
$$

Conjecture (Grothendieck, "hom conjecture"). Let $k$ be finitely generated over $\mathbb{Q}$. If $T / k$ is

Received 4 July 2019, accepted in final form 1 August 2020.

2020 Mathematics Subject Classification 14F35, $11 \mathrm{G} 35$.

Keywords: section conjecture, anabelian geometry.

This journal is (C) Foundation Compositio Mathematica 2021. This article is distributed with Open Access under the terms of the Creative Commons Attribution Non-Commercial License, which permits non-commercial reuse, distribution, and reproduction in any medium, provided that the original work is properly cited. For commercial re-use, please contact the Foundation Compositio Mathematica.

The author is supported by the DFG Priority Program "Homotopy Theory and Algebraic Geometry" SPP 1786. 


\section{G. BRESCIANI}

a smooth variety and $X / k$ is a smooth, proper anabelian variety, then

$$
\operatorname{Hom}_{k}(T, X) \rightarrow \text { Hom-ext }_{\Gamma_{k}}\left(\pi_{1}(T), \pi_{1}(X)\right)
$$

is a bijection.

There is a weaker form of the hom conjecture which restricts the attention to dominant morphisms, and this weaker form has been famously proved by Mochizuki for hyperbolic curves in [Moc99]. We stress that the hom conjecture is strictly stronger: Mochizuki's result only applies to open homomorphisms $\pi_{1}(T) \rightarrow \pi_{1}(X)$, while the hom conjecture regards all homomorphisms.

The second form of the main conjecture is the so-called section conjecture, which is just the hom conjecture for $T=\operatorname{Spec} k$.

Conjecture (Grothendieck, "section conjecture"). Let $k$ be finitely generated over $\mathbb{Q}$. If $X$ is a smooth, proper anabelian variety over $k$, then

$$
X(k) \rightarrow \text { Hom- } \operatorname{ext}_{\Gamma_{k}}\left(\Gamma_{k}, \pi_{1}(X)\right)
$$

is a bijection.

\subsection{A strengthening of the main conjecture}

The set Hom-ext $\Gamma_{k}\left(\Gamma_{k}, \pi_{1}(X)\right)$ has a natural interpretation as the set of isomorphism classes of the category of rational points of the étale fundamental gerbe $\Pi_{X / k}$; see [BV15, $\left.\S 9\right]$. If $X$ is a DM stack, $X(k)$ has the natural structure of a category too (rather than just a set) and Grothendieck's section map extends naturally to a functor

$$
X(k) \rightarrow \Pi_{X / k}(k) .
$$

It is then natural to ask for an equivalence of categories rather than a mere bijection. In fact, already Grothendieck had pointed out that for stacks (which he called "multiplicities"), the correct statement needs an equivalence of categories; see [Gro97, p. 7 of the original letter, p. 55]. For hyperbolic curves, the category structure is known to be trivial (that is, it is just a set) on both sides.

Definition. Let $X$ be a smooth, proper, geometrically connected Deligne-Mumford stack over a field $k$ of characteristic 0 . We say that $X$ is printable (respectively, fundamentally fully faithful, or fff for short) if the natural morphism $X\left(k^{\prime}\right) \rightarrow \Pi_{X / k}\left(k^{\prime}\right)$ is an equivalence (respectively, fully faithful) for every finitely generated extension $k^{\prime} / k$.

The name "printable" is meant to suggest that $\Pi_{X / k}$ "prints" $X$, similarly to how an algebraic space represents a sheaf.

For smooth, proper, hyperbolic curves over a field $k$ that is finitely generated over $\mathbb{Q}$, printability is equivalent to the section conjecture over all finitely generated extensions of $k$; see Proposition 3.7.

\subsection{Results of the paper}

We prove that printability is a geometric property.

Theorem A (Theorem 6.6). Let $k^{\prime} / k$ be a finitely generated extension and $X$ a smooth, proper, geometrically connected DM stack over $k$. Then $X$ is printable (respectively, fff) if and only if $X_{k^{\prime}}$ is printable (respectively, fff). 


\section{GRothendiECK'S ANABELIAN CONJECTURES}

As a consequence, if $k \subseteq \mathbb{C}$ is finitely generated over $\mathbb{Q}$, whether or not $X$ is printable depends only on $X_{\mathbb{C}}$.

We remark that in our proof of Theorem A, the categorical structure is crucial, even for schemes: we do not know whether the same result holds if we replace printability with the analogous statement asking only for a bijection.

The first non-trivial example of expected anabelian DM stacks is that of hyperbolic orbicurves; see [BE14]. There is a natural notion of rational Euler characteristic for orbicurves, and hyperbolic ones are those with negative characteristic.

Conjecture (Borne, Emsalem). Smooth, proper, hyperbolic orbicurves over finitely generated extensions of $\mathbb{Q}$ satisfy the section conjecture.

We prove that hyperbolic orbicurves are fff (in particular, they satisfy the injectivity part of the conjecture) and that the section conjecture for them is equivalent to that for hyperbolic curves.

Theorem B (Theorem 7.2). Let $k$ be finitely generated over $\mathbb{Q}$.

- A smooth, proper orbicurve is fff if and only if its Euler characteristic is less than or equal to 0 .

- If smooth, proper, hyperbolic curves satisfy the section conjecture over every finite extension $k^{\prime} / k$, then the section map is an equivalence for smooth, proper, hyperbolic orbicurves over $k$.

- Smooth, proper, hyperbolic orbicurves are printable if and only smooth, proper, hyperbolic curves are printable.

Thanks to Theorem B and an idea of Borne and Emsalem, we give a new, natural proof of the fact that the section conjecture for proper curves implies the section conjecture for affine curves using orbicurves as an intermediate step; see Theorem 8.1.

We then show that the section conjecture implies the hom conjecture.

Theorem C (Theorem 9.1). Let $X$ be a smooth, proper, geometrically connected DM stack and $T$ a locally noetherian, normal scheme over $k$. Assume that for every $t \in T$, the residue field $k(t)$ is finitely generated over $k$. If $X$ is fff, then $X(T) \rightarrow \Pi_{X}(T)$ is fully faithful. If $X$ is printable, then $X(T) \rightarrow \Pi_{X}(T)$ is an equivalence of categories.

Recall that Grothendieck defined a geometrically connected variety as elementary anabelian if it can be constructed by successive smooth fibrations from hyperbolic curves; see [Gro97]. Merging the concepts of orbicurves and elementary anabelian varieties, in Section 10 we define elementary anabelian stacks. The section conjecture for curves implies that they are printable.

Theorem D (Theorem 10.16). Elementary anabelian stacks over a field $k$ finitely generated over $\mathbb{Q}$ are fff.

If the section conjecture holds for smooth, proper, hyperbolic curves defined over fields that are finitely generated over $\mathbb{Q}$, then elementary anabelian stacks defined over fields that are finitely generated over $\mathbb{Q}$ are printable.

Finally, we highlight two minor results that we think are worth observing. 


\section{G. BRESCIANI}

- If a smooth, proper DM stack $X / k$ is fff, then it has a finite étale cover by an algebraic space; see Proposition 5.1. This suggests that "anabelian" stacks should have a finite étale cover by an algebraic space.

- If a smooth, proper DM stack is printable, then $\pi_{1}\left(X_{\bar{k}}\right)$ has no finite-index abelian subgroups; see Proposition 9.4.

In the appendix, we provide some tools we need which are straightforward generalizations of the work of Borne and Vistoli in [BV15].

\subsection{Conventions and notation}

We always work over a field $k$ of characteristic 0, except in the appendix, where there are no hypotheses on the base field. Curves and orbicurves will always be smooth, geometrically connected and proper, except if we specify differently.

We use underlines to distinguish between sets and sheaves: for instance, Pic is the Picard group, while $\underline{\mathrm{Pic}}$ is the Picard sheaf, or if $X$ is a stack with a rational point $x \in X(k)$, then $\underline{\operatorname{Aut}}_{X}(x)$ is the sheaf of automorphisms of $x$, while $\operatorname{Aut}_{X}(x)=\underline{\operatorname{Aut}}_{X}(x)(k)$.

If $X$ is geometrically connected, we will denote by $\pi_{X}$ the structure morphism $X \rightarrow \Pi_{X / k}$ of the étale fundamental gerbe; see [BV15] and the appendix. If there is no risk of confusion, we may drop the subscript and just write $\pi: X \rightarrow \Pi_{X / k}$. We write $\pi_{1}(X, x)$ for classical étale fundamental groups and $\underline{\pi}_{1}(X, x)=\underline{\mathrm{Aut}}_{\Pi_{X / k}}(\pi(x))$ for étale fundamental group schemes.

Throughout the article (except in Section 8), we restrict our attention to proper $X$. The reason is that the section conjecture is much easier to handle in the proper case, and if one states the anabelian conjectures for DM stacks rather than schemes, then the non-proper case can be recovered from the proper one using a limit process found by Borne and Emsalem (see [BE14, $\S 2.2 .3]$ and Section 8).

There is a small conflict of terminology between two of our major references. For Borne and Vistoli in [BV15], a finite stack over a field $k$ is a stack over $k$ which admits a presentation by a finite groupoid. A finite gerbe is a finite stack which is a gerbe. For the Stacks Project [Sta20], finite morphisms are assumed to be representable. We stick with the Borne-Vistoli terminology.

\section{Stacky going up and going down theorems}

To understand precisely how anabelian geometry for DM stacks should look, the single most important fact to understand is how the section conjecture behaves along finite étale morphisms. In a classical context, that is, for schemes, this situation is well understood and packed in the so-called "going up" and "going down" theorems; see [Sti13, Propositions 110 and 111]. The formalism of étale fundamental gerbes is particularly well suited for the study of this situation: in fact, if $f: Y \rightarrow X$ is a representable, finite étale morphism, then the natural diagram

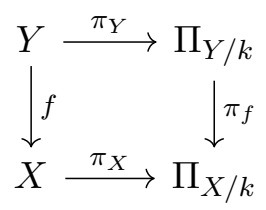

is 2-cartesian; see Proposition A.25. This fact makes the study of finite étale morphism with respect to the section conjecture particularly easy, even for stacks. 


\section{GrothendieCK'S ANABELIAN CONJECTURES}

Proposition 2.1 (Going up). Let $X$ and $Y$ be geometrically connected fibred categories and $f: Y \rightarrow X$ a representable, finite étale morphism. The following are true:

(i) If $X(k) \rightarrow \Pi_{X / k}(k)$ is fully faithful, then $Y(k) \rightarrow \Pi_{Y / k}(k)$ is fully faithful too.

(ii) If $X(k) \rightarrow \Pi_{X / k}(k)$ is an equivalence, then $Y(k) \rightarrow \Pi_{Y / k}(k)$ is an equivalence too.

Proof. This follows directly from the fact that the diagram above is 2-cartesian.

LEMma 2.2 (Extension of the base field). Let $f: A \rightarrow B$ be a morphism of fibred categories over $k$ which are stacks in the étale topology and $L / k$ a finite Galois extension.

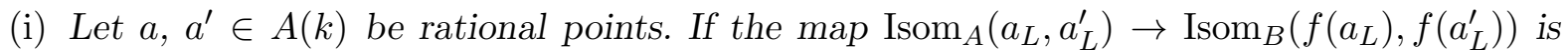
bijective, then the map $\operatorname{Isom}_{A}\left(a, a^{\prime}\right) \rightarrow \operatorname{Isom}_{B}\left(f(a), f\left(a^{\prime}\right)\right)$ is bijective.

(ii) If $A(L) \rightarrow B(L)$ is fully faithful, then $A(k) \rightarrow B(k)$ is fully faithful.

(iii) Let $b \in B(k)$ be a rational point, and suppose that $A(L) \rightarrow B(L)$ is fully faithful. Then $b$ is in the essential image of $A(k) \rightarrow B(k)$ if and only if $b_{L}$ is in the essential image of $A(L) \rightarrow B(L)$.

(iv) If $A(L) \rightarrow B(L)$ is an equivalence, then $A(k) \rightarrow B(k)$ is an equivalence too.

Proof. (i) We have a commutative diagram

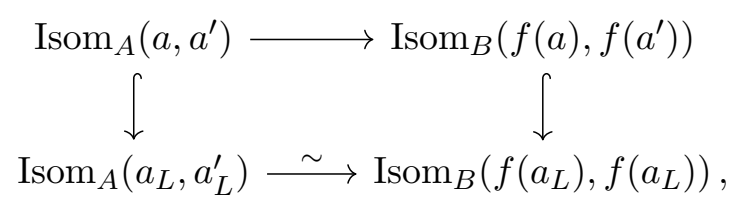

where the vertical arrows are injective and the lower arrow is bijective by hypothesis. Both $A$ and $B$ are stacks in the étale topology; hence, the Isom functors are sheaves and satisfy Galois descent. This means that the sets in the upper row are just the $\operatorname{Gal}(L / k)$-invariant elements of the groups in the lower row. Since the lower horizontal arrow is clearly equivariant, we get that the upper horizontal row is bijective too.

(ii) This follows from part (i).

(iii) The "only if" part is obvious. Now suppose that $b_{L} \simeq f\left(a^{\prime}\right)$ is in the essential image of $A(L) \rightarrow B(L)$. For every $\sigma \in \operatorname{Gal}(L / k)$, we have an isomorphism

$$
\varphi_{\sigma}: \sigma^{*} f\left(a^{\prime}\right) \simeq \sigma^{*} b_{L}=b_{L} \simeq f\left(a^{\prime}\right)
$$

which corresponds to an isomorphism $\psi_{\sigma}: \sigma^{*}\left(a^{\prime}\right) \simeq a^{\prime}$ since $A(L) \rightarrow B(L)$ is fully faithful by hypothesis.

Now we have $\varphi_{\sigma \rho}=\varphi_{\sigma} \circ \sigma^{*} \varphi_{\rho}$ by a direct computation. Since $A(L) \rightarrow B(L)$ is fully faithful, this means that we also have $\psi_{\sigma \rho}=\psi_{\sigma} \circ \sigma^{*} \psi_{\rho}$, and hence by Galois descent, there exists an $a \in A(k)$ such that $a_{L} \simeq a^{\prime}$. Let us check that $f(a) \simeq b$.

We have a chain of isomorphisms

$$
f(a)_{L}=f\left(a_{L}\right) \simeq f\left(a^{\prime}\right) \simeq b_{L} ;
$$

we have to check that this is Galois invariant. This amounts to the fact that, by definition, $f\left(\psi_{\sigma}\right)=\varphi_{\sigma}$.

(iv) This follows from parts (ii) and (iii).

In the following, we will use without mention the fact that if $X$ is a geometrically connected fibred category and $L / k$ is a finite, separable extension, then the natural morphism $\Pi_{X_{L} / L} \rightarrow$ $\Pi_{X / k} \times_{k} L$ is an isomorphism (see Proposition A.18). 


\section{G. BRESCIANI}

Definition 2.3. Let $\mathcal{C}$ and $\mathcal{D}$ be categories, $f: \mathcal{C} \rightarrow \mathcal{D}$ a functor and $p \in \mathcal{C}$ an object. We say that $f$ is fully faithful at $p$ if $\operatorname{Aut}_{\mathcal{C}}(p) \rightarrow \operatorname{Aut}_{\mathcal{D}}(f(p))$ is bijective.

Remark 2.4. Suppose that $\mathcal{C}$ and $\mathcal{D}$ are small categories in which all morphisms are isomorphisms. For example, $X(S)$ has this form for every stack $X$ and every scheme $S$. A functor $f: \mathcal{C} \rightarrow \mathcal{D}$ is fully faithful if and only if it is fully faithful at every point and is injective on isomorphism classes.

Proposition 2.5 (Going down). Let $X$ and $Y$ be geometrically connected fibred categories which are stacks in the étale topology and $f: Y \rightarrow X$ a representable, finite étale morphism. The following are true:

(i) If $Y_{L}(L) \rightarrow \Pi_{Y_{L}}(L)$ is fully faithful for every finite, separable extension $L / k$, then $X(k) \rightarrow$ $\Pi_{X / k}(k)$ is fully faithful.

(ii) If $Y_{L}(L) \rightarrow \Pi_{Y_{L}}(L)$ is an equivalence for every finite, separable extension $L / k$, then $X(k) \rightarrow$ $\Pi_{X / k}(k)$ is an equivalence.

Proof. As in Proposition 2.1, we are going to use the fact that the 2-commutative diagram

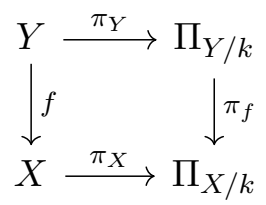

is 2-cartesian; see Proposition A.25. The proof is more complex than that of Proposition 2.1 since now we have to make a descent argument.

(i) First, let us check that $X(k) \rightarrow \Pi_{X / k}(k)$ is fully faithful at every point; next, we will show that it is injective on isomorphism classes.

Full faithfulness at a point. Choose $x \in X(k)$; since $Y \rightarrow X$ is finite étale, there exist a finite Galois extension $L$ and a point $y \in Y_{L}(L)$ such that $f(y) \simeq x_{L}$. Thanks to Lemma 2.2(i), we may suppose $L=k$ and $f(y) \simeq x$. Write

$$
G=\operatorname{Aut}_{\Pi_{X / k}}\left(\pi_{X}(x)\right), \quad H=\operatorname{Aut}_{\Pi_{Y / k}}\left(\pi_{Y}(y)\right) .
$$

Since $Y \rightarrow X$ and $\Pi_{Y / k} \rightarrow \Pi_{X / k}$ are faithful, we have natural embeddings

$$
\pi_{f}: H \subseteq G, \quad f: \operatorname{Aut}_{Y}(y) \subseteq \operatorname{Aut}_{X}(x) .
$$

By an abuse of notation, write $\pi_{X}$ and $\pi_{Y}$ for the homomorphisms $\operatorname{Aut}_{X}(x) \rightarrow G$ and $\operatorname{Aut}_{Y}(y) \rightarrow$ $H$, respectively.

We have an isomorphism $\operatorname{Aut}_{Y}(y) \simeq \operatorname{Aut}_{X}(x) \times_{G} H$, and we also know that $\pi_{Y}: \operatorname{Aut}_{Y}(y) \rightarrow H$ is an isomorphism. In particular, the fact that $\pi_{Y}: \operatorname{Aut}_{Y}(y) \rightarrow H$ is injective implies that $\pi_{X}: \operatorname{Aut}_{X}(x) \rightarrow G$ is injective. Let us prove the surjectivity.

Fix an element $g \in G$. Since the diagram above is 2-cartesian, the triple

$$
\left(x, \pi_{Y}(y), g\right)
$$

gives us a point $y^{\prime} \in Y(k)$ such that $\pi_{Y}\left(y^{\prime}\right) \simeq \pi_{Y}(y)$ and $f\left(y^{\prime}\right) \simeq x$. Since $Y(k) \rightarrow \Pi_{Y / k}(k)$ is fully faithful, there exists an isomorphism $y \rightarrow y^{\prime}$. Because of the 2-cartesianity of the diagram above, the isomorphism $y \rightarrow y^{\prime}$ gives us the following data: two isomorphisms $\alpha: x \rightarrow x, \alpha \in \operatorname{Aut}_{X}(x)$ and $h: \pi_{Y}(y) \rightarrow \pi_{Y}(y), h \in H$ such that

$$
\pi_{f}(h) \circ \mathrm{id}=\pi_{X}(\alpha) \circ g \in G .
$$




\section{GRothendiECK'S ANABELIAN CONJECTURES}

Since $\pi_{Y}: \operatorname{Aut}_{Y}(y) \rightarrow H$ is an isomorphism, there exists a $\beta \in \operatorname{Aut}_{Y}(y)$ such that $\pi_{Y}(\beta)=h$. It follows that $g=\pi_{X}\left(\alpha^{-1} \circ f(\beta)\right)$.

Injectivity on isomorphism classes. Suppose that we have an isomorphism $\alpha: \pi_{X}(x) \rightarrow$ $\pi_{X}\left(x^{\prime}\right)$ for some $x, x^{\prime} \in X(k)$. We want to show that there exists an isomorphism $x \rightarrow x^{\prime}$. Thanks to the preceding point, this is equivalent to showing that $\operatorname{Isom}_{X}\left(x, x^{\prime}\right) \rightarrow \operatorname{Isom}_{\Pi_{X / k}}\left(\pi_{X}(x), \pi_{X}\left(x^{\prime}\right)\right)$ is bijective. There exist a finite Galois extension $L / k$ and a point $y \in Y(L)$ such that $f(y)=x_{L}$. Thanks to Lemma 2.2(i), we may assume $L=k$.

Since

$$
\pi_{f}\left(\pi_{Y}(y)\right)=\pi_{X}(f(y))=\pi_{X}(x) \simeq \pi_{X}\left(x^{\prime}\right)
$$

by 2-cartesianity, there exists a point $y^{\prime} \in Y(k)$ such that $\pi_{Y}\left(y^{\prime}\right) \simeq \pi_{Y}(y)$ and $f\left(y^{\prime}\right) \simeq x^{\prime}$. Now since $Y(k) \rightarrow \Pi_{Y / k}(k)$ is fully faithful by hypothesis and $\pi_{Y}(y) \simeq \pi_{Y}\left(y^{\prime}\right)$, we get an isomorphism $y \simeq y^{\prime}$ which induces an isomorphism $x \simeq x^{\prime}$, as desired.

(ii) This is a direct application of point (i) and Lemma 2.2(iii) together with the observation that every section Spec $k \rightarrow \Pi_{X / k}$ lifts to a section of $\Pi_{Y / k}$ up to a finite, separable field extension: in fact, Spec $k \times_{\Pi_{X / k}} \Pi_{Y / k}$ is a finite étale scheme. To check that Spec $k \times_{\Pi_{X / k}} \Pi_{Y / k}$ is finite étale, observe that up to an extension $k^{\prime} / k$, we have

$$
\text { Spec } k^{\prime} \times_{\Pi_{X / k}} \Pi_{Y / k} \simeq \operatorname{Spec} k^{\prime} \times_{X} Y
$$

for some point Spec $k^{\prime} \rightarrow X$ since $\Pi_{X / k}$ is a gerbe and hence all points are fpqc-locally isomorphic.

In the classical going up and down theorems, there are hypotheses on the so-called centralizers of sections. If $\sigma \in \Pi_{X / k}(k)$ corresponds to a section $s: \operatorname{Gal}(\bar{k} / k) \rightarrow \pi_{1}(X, \bar{x})$, the centralizer of $s$ is the group of elements of $\pi_{1}\left(X_{\bar{k}}, \bar{x}\right)$ centralizing the image of $s$. However, in our results, these hypotheses seem to be absent: the reason is that the notion of centralizer of a section (see [Sti13, $\S 3.3]$ ) fits nicely in our point of view without any additional work. The following Lemma 2.6 explains how.

Lemma 2.6. Let $s: \operatorname{Gal}(\bar{k} / k) \rightarrow \pi_{1}(X, x)$ be a section of the natural projection $\pi_{1}(X, x) \rightarrow$ $\operatorname{Gal}(\bar{k} / k)$ and $C_{s} \subseteq \pi_{1}\left(X_{\bar{k}}, x\right)$ its group of centralizers. Let $\sigma \in \Pi_{X / k}(k)$ be the rational section corresponding to $s$. There is an isomorphism

$$
C_{s} \simeq \underline{\operatorname{Aut}}_{\Pi_{X / k}}(\sigma)(k) .
$$

Proof. This follows from Proposition A.19. Let us explain this.

We have a natural identification

$$
\pi_{1}\left(X_{\bar{k}}, \bar{x}\right)=\underline{\operatorname{Aut}}_{\Pi_{X / k}}(\pi(x))(\bar{k}) .
$$

Since $\Pi_{X / k}$ is a gerbe, there exists an isomorphism $\Phi: \underline{\operatorname{Aut}}_{\Pi_{X / k}}(\pi(x))(\bar{k}) \simeq \underline{\mathrm{Aut}}_{\Pi_{X / k}}(\sigma)(\bar{k})$. The section $s$ induces an action of $\operatorname{Gal}(\bar{k} / k)$ on $\pi_{1}\left(X_{\bar{k}}, x\right)$ by conjugation, and this action coincides with the natural action on $\underline{\operatorname{Aut}}_{\Pi_{X / k}}(\sigma)(\bar{k})$ pulled back to $\underline{\operatorname{Aut}}_{\Pi_{X / k}}(\pi(x))(\bar{k})=\pi_{1}\left(X_{\bar{k}}, x\right)$. Hence, $g \in \pi_{1}\left(X_{\bar{k}}, x\right)$ centralizes $s$ if and only if $\Phi(g) \in \underline{\operatorname{Aut}}_{\Pi_{X / k}}(\sigma)(\bar{k})$ is Galois invariant, in other words, is rational.

\section{Printable DM stacks}

Now that we have established what happens along finite, étale covers, we want to understand what the section conjecture for DM stacks should look like. Clearly, one can just directly trans- 


\section{G. BRESCIANI}

late Grothendieck's section conjecture to DM stacks. Here we hope to show that the right thing to conjecture in general is slightly stronger (but equivalent in the case of hyperbolic curves).

Proposition 3.1. Let $X$ be a proper, smooth, geometrically connected Deligne-Mumford stack over $k$. The following are equivalent:

(i) For every finitely generated extension $k^{\prime} / k$ and for every finite, étale, geometrically connected cover $Y \rightarrow X_{k^{\prime}}$,

$$
Y\left(k^{\prime}\right) \rightarrow \text { Hom-ext }{ }_{\Gamma_{k^{\prime}}}\left(\Gamma_{k^{\prime}}, \pi_{1}(Y)\right)
$$

is bijective (respectively, injective) on isomorphism classes.

(ii) The natural map

$$
X\left(k^{\prime}\right) \rightarrow \Pi_{X / k}\left(k^{\prime}\right)
$$

is an equivalence of categories (respectively, fully faithful) for every finitely generated extension $k^{\prime} / k$.

Proof. Suppose that $X\left(k^{\prime}\right) \rightarrow \Pi_{X / k}\left(k^{\prime}\right)$ is an equivalence (respectively, fully faithful). Then by Proposition A.23, the functor $X_{k^{\prime}}\left(k^{\prime}\right) \rightarrow \Pi_{X_{k^{\prime}} / k^{\prime}}\left(k^{\prime}\right)$ is an equivalence (respectively, fully faithful) too, and hence $Y\left(k^{\prime}\right) \rightarrow$ Hom-ext ${ }_{\Gamma_{k^{\prime}}}\left(\Gamma_{k^{\prime}}, \pi_{1}(Y)\right)$ is bijective (respectively, injective) thanks to the going up theorem Proposition 2.1.

Now suppose that part (i) holds. Let $k^{\prime} / k$ be a finitely generated extension, $x \in X\left(k^{\prime}\right)$ a point and $\pi(x) \in \Pi_{X / k}\left(k^{\prime}\right)$. Write $G=\underline{\operatorname{Aut}}_{\Pi_{X / k}}(\pi(x))$. Since by hypothesis $X\left(k^{\prime}\right) \rightarrow \Pi_{X / k}\left(k^{\prime}\right)$ is bijective (respectively, injective) on isomorphism classes, we only have to show that

$$
\underline{\operatorname{Aut}}_{X}(x) \rightarrow G=\underline{\operatorname{Aut}}_{\Pi_{X / k}}(\pi(x))
$$

induces a bijection on $k^{\prime}$-rational points. Thanks to Proposition A.23, we may suppose $k^{\prime}=k$.

Surjectivity of $\underline{\operatorname{Aut}}_{X}(x)(k) \rightarrow G(k)$. Let $A \subseteq G(k)$ be the image of $\underline{\operatorname{Aut}}_{X}(x)(k)$, and assume for a contradiction that $g \notin A$. Since $A \subseteq G(k)$ is finite and $g \notin A$, there exists a finite-index subgroup $H \subseteq G$ such that $A \subseteq H(k)$ and $g \notin H(k)$ : in order to find it, choose a finite quotient $q: G \rightarrow Q$ such that $q(g) \notin q(A)$, then choose $H$ as the inverse image of $q(A)$. Now consider the 2-fibre product

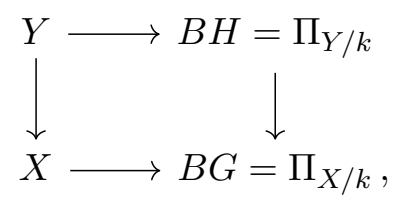

where $B H$ identifies naturally with $\Pi_{Y / k}$. In fact, the universal property of $\Pi_{Y / k}$ gives us a natural map $\Pi_{Y / k} \rightarrow B H$, and thanks to Proposition A.25, the gerbes $\Pi_{Y / k}$ and $B H$ are both subgerbes of $\Pi_{X / k}$ with the same finite index; hence, they coincide.

Now consider $x \in X(k)$ and the distinguished point $d_{H}$ : Spec $k \rightarrow B H$ : by construction, they both map to $\pi(x) \in \Pi_{X / k}(k)$, that is, the distinguished point of $B G$. By the definition of a 2-fibre product, every automorphism of $\pi(x)$ defines a rational point of $Y$ mapping to $x \in X(k)$ and $d_{H} \in B H(k)$. In particular, we have two such rational points $y=\left(x, d_{H}\right.$, id $)$ and $y^{\prime}=\left(x, d_{H}, g\right)$ in $Y(k)$. Let us study the isomorphisms $y \rightarrow y^{\prime}$.

By the definition of a 2-fibre product, an isomorphism $\beta: y=\left(x, d_{H}, \mathrm{id}\right) \rightarrow y^{\prime}=\left(x, d_{H}, g\right)$ is given by a couple of isomorphisms $\alpha: x \rightarrow x, \alpha \in \underline{\operatorname{Aut}}_{X}(x)$ and $h: d_{H} \rightarrow d_{H}, h \in H(k)$ such 


\section{GrothendieCK's ANABELIAN CONJECTURES}

that

$$
h \circ \mathrm{id}=g \circ \pi(\alpha) \in G(k) .
$$

By construction, $\pi(\alpha) \in A \subseteq H(k)$ and clearly $h \in H(k)$; since we have chosen $H$ such that $g \notin H(k)$, the equation above tells us that an isomorphism $y \rightarrow y^{\prime}$ cannot exists; that is, $y$ and $y^{\prime}$ are not isomorphic. But this gives a contradiction since $y$ and $y^{\prime}$ both map to $d_{H} \in B H(k)$ and $Y(k) \rightarrow B H(k)=\Pi_{Y / k}(k)$ is injective on isomorphism classes by hypothesis.

Injectivity of $\underline{\operatorname{Aut}}_{X}(x)(k) \rightarrow G(k)$. Since $\underline{\operatorname{Aut}}_{X}(x)$ is finite étale, up to enlarging the base field we may suppose that $\underline{\operatorname{Aut}}_{X}(x)$ is discrete. Let $A \subseteq G(k)$ be the image of $\underline{\operatorname{Aut}}_{X}(x)(k)$. We can find a finite-index subgroup $H \subseteq G$ such that

$$
H(k) \cap A=\{\operatorname{id}\} \subseteq G(k),
$$

for instance by taking a finite quotient $G \rightarrow Q$ such that $A \rightarrow Q(k)$ is injective and choosing $H$ as the kernel.

Take the 2-fibre product $Y=X \times_{\Pi_{X / k}} B H$ as above, $x \in X(k)$ and the distinguished point $d_{H}:$ Spec $k \rightarrow B H$ to define a rational point $y=\left(x, d_{H}, \mathrm{id}\right) \in Y(k)$. Since

$$
\underline{\operatorname{Aut}}_{Y}(y) \simeq \underline{\operatorname{Aut}}_{X}(x) \times_{G} H
$$

and $A \cap H(k)=\{\mathrm{id}\}$, we get that $\underline{\operatorname{Aut}}_{Y}(y) \subseteq \underline{\operatorname{Aut}}_{X}(x)$ is the kernel of $\pi_{X}: \underline{\operatorname{Aut}}_{X}(x) \rightarrow G$. Hence, we want to prove that $\underline{\operatorname{Aut}}_{Y}(y)$ is trivial.

Suppose for a contradiction that $\underline{\operatorname{Aut}}_{Y}(y)$ is not trivial. A non-trivial, finite étale group scheme is non-special; hence, there exist a finitely generated extension $k^{\prime} / k$ and a point $y^{\prime}:$ Spec $k^{\prime} \rightarrow$ $B \underline{\operatorname{Aut}}_{Y}(y)$ which is not $k^{\prime}$-isomorphic to the distinguished one; that is, $y^{\prime} \not 2 y \in B$ Aut $_{Y}(y)\left(k^{\prime}\right)$.

The morphism $B \underline{\operatorname{Aut}}_{Y}(y) \rightarrow Y$ is the residual gerbe (see [Sta20, Definition 06MU]) of $y$ thanks to [Sta20, Lemmas 0DTI and 06UI]; in particular, it is a monomorphism, and thus $y$ and $y^{\prime}$ define non-isomorphic points of $Y\left(k^{\prime}\right)$. On the other hand, since

$$
\underline{\operatorname{Aut}}_{Y}(y) \rightarrow H=\underline{\operatorname{Aut}}_{\Pi_{Y / k}}\left(\pi_{Y}(y)\right) \subseteq G
$$

is a trivial homomorphism of group schemes, the images of $y$ and $y^{\prime}$ in $B H\left(k^{\prime}\right)=\Pi_{Y / k}\left(k^{\prime}\right)$ are isomorphic. This gives a contradiction since, by hypothesis, $Y\left(k^{\prime}\right) \rightarrow \Pi_{Y / k}\left(k^{\prime}\right)$ is injective on isomorphism classes.

We define printable DM stacks as those satisfying the equivalent conditions of Proposition 3.1.

Definition 3.2. Let $X$ be a smooth, proper, geometrically connected Deligne-Mumford stack. We say that $X$ is printable (respectively, fundamentally fully faithful, or fff for short) if the natural morphism

$$
X\left(k^{\prime}\right) \rightarrow \Pi_{X / k}\left(k^{\prime}\right)
$$

is an equivalence of categories (respectively, fully faithful) for every finitely generated extension $k^{\prime} / k$.

As we will see later, even if this definition seems deeply arithmetic in nature, it is actually purely geometric: if $k \subseteq \mathbb{C}$, the printability of $X$ depends only on $X_{\mathbb{C}}$; see Remark 6.7. This agrees with the ideas expressed by Grothendieck in [Gro97].

Remark 3.3. Extending the definition to Deligne-Mumford stacks seems natural for at least two reasons. One is that moduli stacks of curves are expected to be anabelian, the second is that hyperbolic orbicurves are printable if and only if hyperbolic curves are printable; see Theorem 7.2. We address the question "why not Artin stacks?" in Section 4. 


\section{G. BRESCIANI}

Let us study printability in dimension 0 .

Lemma 3.4. A geometrically connected, geometrically reduced, 0-dimensional DM stack of finite type over $k$ is a finite étale gerbe.

Proof. Let $X$ be such a DM stack. Applying the definition of gerbe, it is immediate to see that $X$ is a gerbe over $k$ if and only if $X_{\bar{k}}$ is a gerbe over $\bar{k}$; hence, we may assume $k$ algebraically closed. Let $U \rightarrow X$ be an étale cover of finite type and $R=U \times_{X} U$; we have that $R \rightrightarrows U$ is a groupoid and the natural map $[U / R] \rightarrow X$ is an isomorphism.

Since $U$ and $R$ are 0-dimensional, reduced schemes of finite type over the algebraically closed field $k$, they are finite disjoint unions of copies of Spec $k$; that is, we may think of them as sets. The groupoid $R \rightrightarrows U$ induces an oriented graph whose set of nodes is $U$ and whose set of arrows is $R$. Let $(U, R)=\left(U_{1}, R_{1}\right) \sqcup \cdots \sqcup\left(U_{n}, R_{n}\right)$ be the connected components of the graph. For every $i$, we have an induced groupoid $R_{i} \rightrightarrows U_{i}$, and, by construction, $X=\bigsqcup_{i}\left[U_{i} / R_{i}\right]$. Since $X$ is connected, it follows that $n=1$.

Now, since the graph induced by $U$ and $R$ is connected, it is immediate to check that $X=$ $[U / R]=B G$, where $G$ is the group of $R$-automorphisms of any point of $U$.

Corollary 3.5. A smooth, proper, geometrically connected DM stack of dimension 0 over $k$ is printable.

Proof. If $X$ is such a DM stack, it is a finite étale gerbe thanks to Lemma 3.4; thus, $X=\Pi_{X / k}$ is obviously printable.

In the following, we show what it means for a scheme to be printable in the classical terms of the section conjecture and of centralizers of sections; see [Sti13, §3.3].

Lemma 3.6. Let $X$ be a smooth, proper, geometrically connected scheme. Then $X$ is printable (respectively, fff) if and only if

- $X_{k^{\prime}}$ satisfies the section conjecture (respectively, the injectivity part of the section conjecture) for every finitely generated extension $k^{\prime} / k$, and

- for every $x \in X\left(k^{\prime}\right)$, the associated section in Hom- $\operatorname{ext}_{\Gamma_{k^{\prime}}}\left(\Gamma_{k^{\prime}}, \pi_{1}(X)\right)$ has trivial centralizer.

Proof. As we have shown in Lemma 2.6, the automorphism groups of the points of the fundamental gerbe correspond to centralizers of sections of the étale fundamental group. Hence, if $X$ is a scheme, asking for an equivalence of categories corresponds to asking for a bijection on isomorphism classes together with the triviality of centralizers.

Proposition 3.7. Let $k$ be finitely generated over $\mathbb{Q}$.

- Smooth proper curves over $k$ are fundamentally fully faithful if and only if they have positive genus.

- Hyperbolic curves over $k$ are printable if and only if they satisfy the section conjecture over every finitely generated extension of the base field.

Proof. For smooth, proper curves with Euler characteristic less than or equal to 0, centralizers of sections coming from rational points are trivial, thanks either to [Sti13, Propositions 36 and 104] or to the full faithfulness part of Proposition 3.1. Apply Lemma 3.6.

Proposition 3.8. Let $Y$ and $X$ be smooth, proper, geometrically connected DM stacks over a field $k$ and $Y \rightarrow X$ a finite étale covering. Then $X\left(k^{\prime}\right) \rightarrow \Pi_{X}\left(k^{\prime}\right)$ is an equivalence (respectively, 


\section{GrothendiecK's AnABELIAN CONJECTURES}

fully faithful) for every finite, separable extension $k^{\prime} / k$ if and only if the same holds for $Y$. In particular, $Y$ is printable (respectively, fff) if and only if $X$ is printable (respectively, fff).

Proof. This is a straightforward application of the going up and down theorems, Propositions 2.1 and 2.5.

\section{Why not Artin stacks}

One may wonder: why DM stacks and not algebraic (that is, Artin) stacks? The answer is based on one's taste. DM stacks seem more natural since $\Pi_{X / k}$ is profinite étale and Proposition 3.1 fails for algebraic stacks. For example, if $G$ is a connected algebraic group, then condition (i) of Proposition 3.1 holds for $B G$ if and only if $G$ is special, while condition (ii) if and only if $G$ is trivial. Hence, it makes a difference if we choose condition (i) or (ii) as definition of printability for algebraic stacks.

If we choose conditions (i), we should for instance consider $B \mathrm{GL}_{n}$ as printable even if $B \mathrm{GL}_{n} \rightarrow \Pi_{B \mathrm{GL}_{n}}=$ Spec $k$ is not an equivalence of categories on rational points, and this does not seem very pleasant. On the other hand, if we choose condition (ii), the following proposition shows that we get back to DM stack.

Proposition 4.1. Let $X$ be a separated, geometrically connected algebraic stack that is locally of finite type over $k$. Suppose that

$$
X\left(k^{\prime}\right) \rightarrow \Pi_{X / k}\left(k^{\prime}\right)
$$

is fully faithful for every finitely generated extension $k^{\prime} / k$. Then $X$ is a DM stack.

Proof. Since we are in characteristic 0, it is enough to show that $\underline{A u t}_{X}(x)$ is finite for any geometric point $x$; see [Sta20, Lemma 0DSN]. Since $X$ is locally of finite type, we may assume that $x$ is defined over a finitely generated extension $k^{\prime} / k$. Thanks to Proposition A.23, we may suppose $k^{\prime}=k$; that is, $x \in X(k)$ is a rational point. Since $X$ is separated, $\underline{\operatorname{Aut}}_{X}(x)$ is a group scheme of finite type; see [Sta20, Lemma 0DTS].

Let $\pi(x) \in \Pi_{X / k}(k)$ be the image of $x$; we have a homomorphism of group schemes

$$
\underline{\mathrm{Aut}}_{X}(x) \stackrel{\pi}{\rightarrow} \underline{\mathrm{Aut}}_{\Pi_{X / k}}(\pi(x)) .
$$

This homomorphism has trivial kernel: otherwise, since $\underline{\mathrm{Aut}}_{X}(x)$ is of finite type, up to enlarging the base field we may suppose that there exists a rational point $\varphi \in \operatorname{ker}(\pi)(k)$ different from the identity. But $\underline{\mathrm{Aut}}_{X}(x) \rightarrow \underline{\mathrm{Aut}}_{\Pi_{X / k}}(\pi(x))$ is injective on rational points by hypothesis, we are in characteristic 0, and hence $\operatorname{ker}(\pi)$ is trivial. By the following Lemma 4.2, we get that $\underline{\operatorname{Aut}}_{X}(x)$ is finite.

Lemma 4.2. Let $f: G \rightarrow P$ be a homomorphism of group schemes over a field $k$ with trivial kernel. Assume that $G$ is of finite type and $P$ is profinite. Then $G$ is finite.

Proof. We may base change everything to $\bar{k}$ and assume that $k$ is algebraically closed. Let $g \in G$ be any point with residue field $k(g)$. Since $G$ is of finite type, there exists an irreducible variety $U$ with function field $k(U)=k(g)$ and a locally closed embedding $U \subseteq G$. Since $P$ is profinite and $k$ is algebraically closed, $f(g)$ is rational; thus, by construction, all the points of $U(k) \subseteq G(k)$ map to $f(g)$. Since $G \rightarrow P$ has trivial kernel, it follows that $U(k)$ has only one point and hence $k(g)=k(U)=k$. Since $G$ is of finite type and every point is rational, it is finite. 


\section{G. BRESCIANI}

\section{Covers by algebraic spaces}

It turns out that fff DM stacks must have a non-obvious topological feature: they are uniformizable in the sense of Noohi; that is, they have a finite étale cover by an algebraic space; see [Noo04, Definition 6.1]. Noohi essentially proves this in [Noo04, Theorem 6.2], but the connection with the section conjecture is not stated in his work.

Rather than using Noohi's result, we prove it again in our setting: a formal comparison with Noohi's theory would be longer. The idea behind the proof is essentially the same.

Proposition 5.1. Let $X$ be a geometrically connected, separated DM stack of finite type over $k$, and suppose that the natural morphism

$$
X\left(k^{\prime}\right) \rightarrow \Pi_{X / k}\left(k^{\prime}\right)
$$

is faithful for every finitely generated $k^{\prime} / k$.

There exist a finite étale gerbe $\Phi$ with a representable morphism $X \rightarrow \Phi$ and a finite étale cover $E \rightarrow X$ with $E$ an algebraic space. If $\Pi_{X / k}(k) \neq \emptyset$, we can choose $E$ to be geometrically connected.

Proof. Let $\xi:$ Spec $k^{\prime} \rightarrow X$ be the generic point of an irreducible component of $X$ with $k^{\prime}$ finitely generated over $k$. Up to enlarging $k^{\prime}$, we may assume that $\underline{A u t}_{X}(\xi)$ is discrete over $k^{\prime}$. By hypothesis, there exists a finite étale gerbe $\Phi_{0}$ over $k$ with a morphism $\varphi_{0}: X \rightarrow \Phi_{0}$ which is faithful at $\xi$; that is, $\underline{\operatorname{Aut}}_{X}(\xi) \rightarrow \underline{\operatorname{Aut}}_{\Phi_{0}}\left(\varphi_{0}(\xi)\right)$ is injective.

Consider the relative inertia $I_{X / \Phi_{0}}$. By generic flatness, there exists an open, irreducible subset $U_{0} \subseteq X$ such that $\xi \in U_{0}$ and the restriction of $I_{X / \Phi_{0}} \rightarrow X$ to $U_{0 \text {,red }} \subseteq X_{\text {red }}$ is flat. Since $I_{X / \Phi_{0}} \rightarrow X$ is proper and unramified too, its restriction to $U_{0 \text {,red }}$ is finite étale. Since $X \rightarrow \Phi_{0}$ is faithful at $\xi$, it moreover follows that the restriction of $I_{X / \Phi_{0}}$ to $U_{0 \text {,red }}$ is a finite étale morphism of degree 1, that is, an isomorphism. In particular, $I_{U_{0} / \Phi_{0}} \rightarrow U_{0}$ is radicial and unramified, thus a monomorphism. Moreover, it has a section; hence, it is an isomorphism, and hence $U_{0} \rightarrow \Phi_{0}$ is faithful.

Now let $X_{1}=X \backslash U_{0}$. We may repeat the process and find $U_{1} \subseteq X_{1}$ with a finite étale gerbe $\Phi_{1}$ and a morphism $X \rightarrow \Phi_{1} \rightarrow \Phi_{0}$ such that $U_{1} \rightarrow \Phi_{1}$ is faithful, define $X_{2}=X_{1} \backslash U_{1}$ and continue by recursion. By noetherian descent, the process ends; thus, for some large $N$, we have that $X_{N+1}$ is empty and $X \rightarrow \Phi_{N}$ is faithful. Choose $\Phi=\Phi_{N}$. We have that $X \rightarrow \Phi$ is representable since a faithful morphism of algebraic stacks is representable; see [Sta20, Lemma 04Y5].

In order to find $E$, observe that since $\Phi$ is a finite étale gerbe, there exist a finite, separable extension $k^{\prime} / k$ and a section $\operatorname{Spec} k^{\prime} \rightarrow \Phi$. Take $E=\operatorname{Spec} k^{\prime} \times_{\Phi} X$.

Suppose $\Pi_{X / k}(k) \neq \emptyset$; in particular, we have a section Spec $k \rightarrow \Phi$. Thanks to [BV15, Lemma 5.12], we may assume that $X \rightarrow \Phi$ is Nori-reduced: this exactly means that $E=\operatorname{Spec} k \times_{\Phi} X$ is geometrically connected; see [BV15, Remark 5.11]. We remark that in [BV15, Remark 5.11], the authors assume that $X$ is geometrically reduced, a hypothesis we do not have, but it can be checked that they do not actually use it (provided that in their proof, algebraic closures are replaced by separable closures).

\section{Printability depends only on the geometric type}

A priori, our definition of printable DM stack depends on the base field $k$. It turns out that it is actually independent of it. 


\section{GrothendiecK's AnABELIAN CONJECTURES}

In order to prove this, we have to generalize some Galois-theoretic facts about finite extensions of fields to finitely generated extensions. We work in characteristic 0 in order to avoid inseparability issues.

Lemma 6.1. Let $k^{\prime} / h / k$ be finitely generated extensions of a field $k$ of characteristic 0 . Let $\sigma$ be a non-trivial automorphism of $h$ of finite order which is trivial on $k$. Then there exists a finite extension $k^{\prime \prime} / k^{\prime}$ such that $\sigma$ extends to an automorphism of finite order of $k^{\prime \prime}$.

Proof. Up to replacing $k$ with the subfield of $h$ fixed by $\sigma$, we may assume that $h / k$ is finite.

Let $t_{1}, \ldots, t_{n}$ be a transcendence basis of $k^{\prime} / h$. We have that $\sigma$ extends to an automorphism of $h\left(t_{1}, \ldots, t_{n}\right)$ that is trivial on $k\left(t_{1}, \ldots, t_{n}\right)$ by acting trivially on $t_{i}$. Since $h$ is finite over $k$, the extension $k^{\prime} / k\left(t_{1}, \ldots, t_{n}\right)$ is finite. Choose a Galois closure $k^{\prime \prime} / k^{\prime} / k\left(t_{1}, \ldots, t_{n}\right)$; the automorphism of $h\left(t_{1}, \ldots, t_{n}\right)$ extends to $k^{\prime \prime}$.

LEMma 6.2. Let $k^{\prime} / h / k$ be finitely generated extensions of a field $k$ of characteristic 0 . If $h / k$ is non-trivial, then there exists a finitely generated extension $k^{\prime \prime} / k^{\prime} / k$ with an automorphism $\sigma: k^{\prime \prime} \rightarrow k^{\prime \prime}$ of finite order which is trivial on $k$ but non-trivial on $h$.

Proof. Let us do this in three cases.

Case 1: $k^{\prime}=h$ and $k$ is not algebraically closed in $h$. Let $l=\bar{k}^{h}$, fix a Galois closure $\tilde{l}$ of $l$, and set $\widetilde{h}=\widetilde{l} \otimes_{l} h$. Since $l$ is algebraically closed in $h$, we get that $\widetilde{h}$ is an integral domain; moreover, it is a field since it is finite over $h$. Now take any automorphism $\sigma$ of $\widetilde{l} / k$ which is non-trivial on $h \cap l$ (it exists by Galois theory since the extension $h \cap l / k$ is non-trivial) and apply Lemma 6.1.

Case 2: $k^{\prime}=h$ and $k$ is algebraically closed in $h$. Let $k^{\prime \prime}$ be the fraction field of the integral domain $h \otimes h$, and let $\sigma$ be the automorphism of $h \otimes h$ which permutes the two coordinates: this extends to the fraction field $k^{\prime \prime}$, and thus we conclude.

General case. By the preceding cases, there exists a finitely generated extension $h^{\prime} / h$ with an automorphism $\sigma$ of $h^{\prime}$ that is trivial on $k$ but not trivial on $h$. Choose any finitely generated extension $k^{\prime \prime} / k$ which contains both $h^{\prime}$ and $k^{\prime}$ as subextensions. Thanks to Lemma 6.1, there exists a further finite extension $k^{\prime \prime \prime} / k^{\prime \prime}$ such that $\sigma$ extends to $k^{\prime \prime \prime}$.

Lemma 6.3. Let $G$ be a profinite étale group scheme over $k$, and suppose that

$$
G\left(k^{\prime}\right)=\{\mathrm{id}\}
$$

for every field $k^{\prime}$ finite over $k$. Let $T \rightarrow$ Spec $k$ be a $G$-torsor and $k^{\prime} / k$ a finitely generated extension such that $T_{k^{\prime}} \rightarrow$ Spec $k^{\prime}$ is the trivial torsor. Then $T$ is trivial.

Proof. Let $p \in T$ be the image of a point Spec $k^{\prime} \rightarrow T$; then $k(p) / k$ is finite and separable since $k^{\prime} / k$ is finitely generated and $T \rightarrow$ Spec $k$ is profinite étale. Let $\overline{k(p)}$ be a Galois closure of $k(p) / k$. We have that $\operatorname{Spec} k(p) \otimes_{k} \widetilde{k(p)} \rightarrow T_{\widehat{k(p)}}$ is a closed embedding because it is the base change of $\operatorname{Spec} k(p) \rightarrow T$, which is a closed embedding. But if $k(p) / k$ is non-trivial, $\operatorname{Spec} k(p) \otimes_{k} \widetilde{k(p)}$ is a finite étale scheme with more than one point; hence, we get a contradiction because $T_{\widehat{k(p)}} \simeq$ $G_{\widetilde{k(p)}}$ has only one rational point by hypothesis.

Example 6.4. Let $X / k$ be an fff algebraic space and $p \in X(k)$ a rational point. Then

$$
\underline{\pi}_{1}(X, x)=\underline{\operatorname{Aut}}_{\Pi_{X / k}}(\pi(x))
$$

respects the hypothesis of Lemma 6.3 by the definition of fff. In classical terms, the fact that $\underline{\pi}_{1}(X, x)$ has no non-trivial rational points amounts to the triviality of centralizers of $\pi(x)$; see Lemma 2.6. 


\section{G. BRESCIANI}

Lemma 6.5. Let $k^{\prime} / k$ be a finitely generated extension of the base field and $X$ a separated, fff DM stack of finite type over $k$. Let $s \in \Pi_{X / k}(k)$ be such that $s^{\prime}=s_{k^{\prime}}$ is in the essential image of $X\left(k^{\prime}\right) \rightarrow \Pi_{X / k}\left(k^{\prime}\right)$. Then $s$ is in the essential image of $X(k) \rightarrow \Pi_{X / k}(k)$.

Proof. If $k^{\prime} / k$ is finite, then since we are in characteristic 0 , we may extend $k^{\prime} / k$ and suppose that it is Galois. The statement is then the content of Lemma 2.2(iii); hence, we may freely take finite extensions of the base field.

Thanks to Proposition 5.1, we may take a finite cover $E \rightarrow X$ with $E$ an algebraic space. Up to taking a finite extension of the base field and by taking connected components, we may suppose that $E$ is geometrically connected. Since every point of $X$ extends to $E$ up to a finite extension, by an easy diagram chasing, we see that we may replace $X$ with $E$ and suppose that $X$ is an algebraic space.

Now, by hypothesis, we have a point $x^{\prime} \in X\left(k^{\prime}\right)$ which maps to $s^{\prime} \in \Pi_{X / k}\left(k^{\prime}\right)$. Since $X$ is separated, $x^{\prime}$ has a residue field $k\left(x^{\prime}\right)$. If the extension $k\left(x^{\prime}\right) / k$ is non-trivial, then thanks to Lemma 6.2, there exists a finitely generated extension $k^{\prime \prime} / k^{\prime} / k\left(x^{\prime}\right) / k$ with a non-trivial automorphism $\sigma: k^{\prime \prime} \rightarrow k^{\prime \prime}$ which fixes $k$ but does not fix $k\left(x^{\prime}\right)$. In particular, $\sigma^{*} x_{k^{\prime \prime}}^{\prime} \neq x_{k^{\prime \prime}}^{\prime}$, but

$$
\pi_{X}\left(\sigma^{*} x_{k^{\prime \prime}}^{\prime}\right)=\sigma^{*} \pi_{X}\left(x_{k^{\prime \prime}}^{\prime}\right)=\sigma^{*} s_{k^{\prime \prime}}^{\prime}=s_{k^{\prime \prime}}^{\prime}=\pi_{X}\left(x^{\prime}\right) \in \Pi_{X / k}\left(k^{\prime \prime}\right)
$$

because $s^{\prime}$ is defined on $k$. This gives a contradiction since $X$ is fff by hypothesis. Hence, $k\left(x^{\prime}\right)=k$; that is, there exists an $x \in X(k)$ with $x_{k^{\prime}}=x^{\prime}$.

We now want to show that $\pi_{X}(x)=s$ using the fact that $\pi_{X}(x)_{k^{\prime}}=\pi_{X}\left(x_{k^{\prime}}\right)=\pi_{X}\left(x^{\prime}\right)=s_{k^{\prime}}$. We may think of $s$ as a $\underline{\pi}_{1}(X, x)=\underline{\operatorname{Aut}}_{\Pi_{X / k}}(x)$-torsor, $\pi_{X}(x)$ is the trivial $\underline{\pi}_{1}(X, x)$-torsor, and $k^{\prime}$ splits the torsor $s$. Then $s$ is trivial because $\underline{\pi}_{1}(X, x)$ respects the hypothesis of Lemma 6.3 since $X$ is fff.

TheOREM 6.6. Let $k^{\prime} / k$ be a finitely generated extension and $X$ a smooth, proper, geometrically connected DM stack over $k$. Then $X$ is printable (respectively, fff) if and only if $X_{k^{\prime}}$ is printable (respectively, fff).

Proof. We only do this for printability, the argument for fff is analogous.

If $X$ is printable, $X_{k^{\prime}}$ is printable by definition since $\Pi_{X_{k^{\prime}} / k^{\prime}}=\Pi_{X / k} \times_{k} k^{\prime}$ thanks to Proposition A.23.

On the other hand, suppose that $X_{k^{\prime}}$ is printable. If $k^{\prime} / k$ is finite, up to a finite extension, we may suppose that it is Galois too. Then this is the content of Lemma 2.2.

Now that we can take arbitrary finite extensions of the base field, we may reduce to the case in which $X$ is an algebraic space using the same argument we have used in Lemma 6.5.

Hence, we may suppose that $X$ is an algebraic space. Let $L / k$ be a finitely generated extension; we want to show that $X(L) \rightarrow \Pi_{X / k}(L)$ is an equivalence. There exists a finitely generated extension $L^{\prime}$ of $k^{\prime}$ containing $L$; up to taking extensions, we may suppose $L=k$ and $L^{\prime}=k^{\prime}$.

First, we must show that $\pi_{X}: X(k) \rightarrow \Pi_{X / k}(k)$ is fully faithful. Since $X$ is an algebraic space, this amounts to showing injectivity on isomorphism classes together with the fact that for every $x \in X(k)$, the group $\underline{\operatorname{Aut}}_{\Pi_{X / k}}\left(\pi_{X}(x)\right)(k)$ is trivial. But these are direct consequences of the analogous facts over $k^{\prime}$, which are true by hypothesis.

Finally, we have to show the essential surjectivity of $\pi_{X}: X(k) \rightarrow \Pi_{X / k}(k)$; this is the content of Lemma 6.5.

Remark 6.7. Thanks to Theorem 6.6, we can see printability as a geometric property, rather than an arithmetic one, and this is coherent with Grothendieck's ideas. If a DM stack $X$ is defined 


\section{GRothendiECK'S ANABELIAN CONJECTURES}

over a subfield $k$ of $\mathbb{C}$ which is finitely generated over $\mathbb{Q}$, then whether or not $X$ is printable depends only on $X_{\mathbb{C}}$; that is, printability is a geometric notion. Clearly this is a tautology; we are not really able to describe in purely geometrical terms which DM stacks over $\mathbb{C}$ descend to printable DM stacks: still, we think it is worth observing that the arithmetic property depends only on the geometry of the variety.

\section{Orbicurves}

The first non-trivial example of expected anabelian DM stacks is that of hyperbolic orbicurves. Borne and Emsalem conjectured [BE14, Conjecture 2] that the section conjecture holds for them.

A proper orbicurve is a smooth, proper DM stack of dimension 1 which is generically a scheme. In order to be clear, we will use Fraktur letters for orbicurves and normal ones for schemes.

Let $\mathfrak{X}$ be an orbicurve with coarse moduli space $\mathfrak{X} \rightarrow X$. There exists a maximal open subset $U \subseteq \mathfrak{X}$ which is a scheme and for which $U \rightarrow X$ is an open immersion. Let $D=X \backslash U$, and define the rational Euler characteristic of $\mathfrak{X}$ as

$$
\chi(\mathfrak{X})=2-2 g-\sum_{x \in D} \frac{r_{x}-1}{r_{x}}[k(x): k],
$$

where $r_{x}$ is the degree of the residual gerbe at $x$, that is, the ramification degree of $\mathfrak{X} \rightarrow X$ at $x$. The orbicurve $\mathfrak{X}$ is hyperbolic (respectively, elliptic, parabolic) if $\chi(\mathfrak{X})<0$ (respectively, $\chi(\mathfrak{X})=0, \chi(\mathfrak{X})>0)$; see [BE14, §2.2], [BN06, Proposition 5.11].

If $\mathfrak{Y} \rightarrow \mathfrak{X}$ is a finite étale cover of degree $d$, the Riemann-Hurwitz formula applied to the associated (possibly ramified) morphism of coarse moduli spaces $Y \rightarrow X$ yields the usual formula

$$
\chi(\mathfrak{Y})=d \chi(\mathfrak{X}) .
$$

Proposition 7.1. Let $\mathfrak{X}$ be an orbicurve with coarse moduli space $\mathfrak{X} \rightarrow X, U \subseteq \mathfrak{X}$ a maximal open subset which is scheme, $D=X \backslash U$, and $r_{x}$ the degree of the residual gerbe at $x$ for $x \in D$. Suppose that we are not in one of the following cases:

$-g(X)=0, \operatorname{deg} D=1$

- $g(X)=0, D=\left\{x_{1}, x_{2}\right\}, x_{i} \in X(k)$ rational, $r_{x_{1}} \neq r_{x_{2}}$.

Then there exist a finite extension $k^{\prime} / k$ and a smooth, geometrically connected curve $Y$ over $k^{\prime}$ with a geometrically Galois finite étale cover $Y \rightarrow \mathfrak{X}_{k^{\prime}}$. If $\Pi_{\mathfrak{X} / k}(k) \neq \emptyset$, we may furthermore assume $k^{\prime}=k$.

Proof. Since everything is of finite type, with standard arguments we can obtain the general case once we know that the proposition is true for $k$ finitely generated over $\mathbb{Q}$. Now we may fix an embedding $k \subseteq \mathbb{C}$, and thus reduce to $k=\mathbb{C}$. For $k=\mathbb{C}$, this is [BN06, Proposition 5.7].

We are able to work with orbicurves because those with non-positive Euler characteristic have a finite étale covering which is a curve; that is, they are uniformizable in the sense of Noohi. This is not only a useful feature, but a necessary one: thanks to Propositions 3.1 and 5.1, it is implied by the injectivity part of the section conjecture for orbicurves. It is rather remarkable that this necessary topological feature happens to be true.

TheOREM 7.2. Let $k$ be finitely generated over $\mathbb{Q}$.

- A smooth, proper orbicurve $\mathfrak{X}$ is fundamentally fully faithful if and only if $\chi(\mathfrak{X}) \leqslant 0$. 


\section{G. BRESCIANI}

- If smooth, proper, hyperbolic curves satisfy the section conjecture over every finite extension $k^{\prime} / k$, then the section map is an equivalence for smooth, proper, hyperbolic orbicurves over $k$.

- In particular, smooth, proper, hyperbolic orbicurves are printable if and only if smooth, proper, hyperbolic curves are printable.

Proof. Thanks to Propositions 3.7 and 3.8, Theorem 6.6 and Proposition 7.1, we may reduce to one of the following cases: $\mathfrak{X}$ is either a curve or a simply connected orbicurve. Both these cases are obvious.

COROLlary 7.3. The section conjecture holds for hyperbolic orbicurves if and only if it holds for hyperbolic curves.

\section{Affine curves}

There is a version of the section conjecture for affine curves. If $U$ is a smooth, geometrically connected curve with smooth completion $X$ and complement $D=X \backslash U$, every "missing" rational point $x \in D(k)$ defines a so-called packet of cuspidal sections $\mathcal{P}_{x} \subseteq \Pi_{U / k}(k)$; see [EH08] and [Sti12]. Following [Sti12], the packet based at the cusp $x$ is defined as follows. Let $\mathcal{O}_{X, x}^{\mathrm{h}}$ be the henselianization of the local ring, and define the scheme of nearby points $U^{x}=U \times{ }_{X} \operatorname{Spec}\left(\mathcal{O}_{X, x}^{\mathrm{h}}\right)$. The packet $\mathcal{P}_{x}$ is then defined as the image of

$$
\Pi_{U^{x} / k}(k) \rightarrow \Pi_{X / k}(k) .
$$

The section conjecture for $U$ says that if $k$ is finitely generated over $\mathbb{Q}$ and $U$ has negative Euler characteristic, then the section map

$$
U(k) \sqcup \bigsqcup_{x \in D(k)} \Pi_{U^{x} / k}(k) \rightarrow \Pi_{U / k}(k)
$$

is bijective on isomorphism classes.

As showed by Borne and Emsalem in [BE14, §2.2.3], the section conjecture for orbicurves implies easily the section conjecture for affine curves. If we put together their observation and Theorem 7.2, we obtain a new proof of the following classical result; see [Sti13, Propositions 103 and 250].

THEOREM 8.1. The section map is injective for affine hyperbolic curves. The section conjecture for proper hyperbolic curves implies the section conjecture for affine hyperbolic curves.

Let us show how the ideas of Borne and Emsalem fit nicely in our formalism, giving a clear picture of packets of tangential points and of the section conjecture for affine curves.

Let $U, X, D$ be as above, and define $U_{n}$ as the root stack supported over $X$ with ramification of degree $n$ along the divisor $D$; see [AGV08, Appendix B.2] and [BE14, $\S 2.2]$ for the definition of a root stack. The stack $U_{n}$ is an orbicurve with coarse moduli space $U_{n} \rightarrow X$ such that $U \subseteq X$ is the schematic locus of $U_{n}$, and $U_{n} \rightarrow X$ has ramification index equal to $n$ at each point over $D$. Define

$$
\widehat{U}={\underset{\lim }{n}}_{n} U_{n}
$$

as the projective limit: it is an fpqc stack with natural morphisms $U \hookrightarrow \widehat{U}$ and $\widehat{U} \rightarrow X$. The pro-algebraic stack $\widehat{U}$, called the infinite root stack, can also be constructed using logarithmic geometry; see [TV18] for details. 


\section{GrothendiECK'S ANABELIAN CONJECTURES}

The natural morphism

$$
\Pi_{U / k} \rightarrow \Pi_{\widehat{U} / k} \simeq \underbrace{\lim }_{n} \Pi_{U_{n} / k}
$$

is an isomorphism; this is proved in [Bor09, Proposition 3.2.2]. In view of this fact, from our point of view one could simply consider $\widehat{U}$ as a "complete substitute" of $U$ and decide that the section conjecture for $U$ is the section conjecture for $\widehat{U}$. Let us show that this coincides with the classical approach using packets.

Fix a rational point $x \in D(k)$. Using Abhyankar's lemma, the étale fundamental gerbe $\Pi_{U^{x} / k}$ of the scheme of nearby points $U^{x}$ can be easily computed to be abelian and banded by $\widehat{\mathbb{Z}}(1)$. Let $\bar{x}$ be the closed point of $\operatorname{Spec}\left(\mathcal{O}_{X, x}^{\mathrm{h}}\right)$; if we consider the infinite root stack $\widehat{U}^{x}$ of $\operatorname{Spec}\left(\mathcal{O}_{X, x}^{\mathrm{h}}\right)$ at $\bar{x}$, it is immediate to check that the structure map $\widehat{U}^{x} \rightarrow \Pi_{\widehat{U}^{x} / k}=\Pi_{U^{x} / k}$ induces an isomorphism between the residue gerbe $\widehat{U}^{x} \times_{\operatorname{Spec}\left(\mathcal{O}_{X, x}^{h}\right)} \bar{x}=\widehat{U}_{\bar{x}}^{x}$ and $\Pi_{U^{x} / k}$. This gives us a map

$$
\Pi_{U^{x} / k}=\widehat{U}_{\bar{x}}^{x} \rightarrow \widehat{U}_{x}=\widehat{U} \times_{X} x,
$$

which is easily checked to be an isomorphism. It follows that the packet at $x$ identifies naturally with the isomorphism classes of rational points of $\widehat{U}$ over $x$.

Proof of Theorem 8.1. If $U$ is hyperbolic, $\chi\left(U_{n}\right)<0$ for $n$ big enough. Hence $U_{n}(k) \rightarrow \Pi_{U_{n} / k}(k)$ is fully faithful for $n$ big enough, and passing to the limit shows that the same is true for $\widehat{U}$. If the section conjecture holds for proper hyperbolic curves, the section map is an equivalence for $U_{n}$ thanks to Theorem 7.2. Passing to the limit, we see that the section map is an equivalence for $\widehat{U}$, and thus the section conjecture holds for $U$.

\section{The section conjecture implies the hom conjecture}

If $X$ is printable, we expect the functor

$$
X(T) \rightarrow \Pi_{X / k}(T)
$$

to be an equivalence for a much larger class than finitely generated extensions of $k$. At least, we should have smooth schemes: we actually show that normality together with a broad finiteness condition is enough.

Recall that for $X$ a hyperbolic curve and $T$ smooth, Mochizuki proved in [Moc99, Theorem A] that $X(T) \rightarrow \Pi_{X / k}(T)$ induces a bijection between dominant morphisms $T \rightarrow X$ and sections $T \rightarrow \Pi_{X / k}$ inducing an open homomorphism of étale fundamental groups. Here, we are concerned with all sections $T \rightarrow \Pi_{X / k}$ : the hom conjecture implies that if $T$ is geometrically connected, a section $T \rightarrow \Pi_{X / k}$ either induces an open homomorphism of étale fundamental groups or factors through $\operatorname{Spec} k$, but no proof of this is known.

Theorem 9.1. Let $X$ be a smooth, proper, geometrically connected DM stack and $T$ a locally noetherian, normal scheme over $k$. Assume that for every $t \in T$, the residue field $k(t)$ is finitely generated over $k$. If $X$ is fff, then $X(T) \rightarrow \Pi_{X}(T)$ is fully faithful. If $X$ is printable, then $X(T) \rightarrow \Pi_{X}(T)$ is an equivalence of categories.

Proof. Since a locally noetherian, normal scheme is a disjoint union of integral normal schemes by [Sta20, Lemma 033N], we may assume that $T$ is integral.

Full faithfulness. Let $t_{1}, t_{2}: T \rightarrow X$ be two morphisms, $\pi\left(t_{1}\right), \pi\left(t_{2}\right)$ their images in $\Pi_{X / k}(T)$ and $\left(t_{1}, t_{2}\right) \in X \times X(T)$. We have that $\underline{\operatorname{Isom}}_{X}\left(t_{1}, t_{2}\right)$ is proper, unramified and hence finite 


\section{G. BRESCIANI}

over $T$ since $X$ is separated and DM. Moreover, $\Pi_{X / k}$ is a projective limit of DM, separated stacks; hence, for the same reason, $\underline{\operatorname{Isom}}_{\Pi_{X / k}}\left(\pi\left(t_{1}\right), \pi\left(t_{2}\right)\right)$ is profinite over $T$.

Since $\underline{\operatorname{Isom}}_{X}\left(t_{1}, t_{2}\right)$ and $\underline{\operatorname{Isom}}_{\Pi_{X / k}}\left(\pi\left(t_{1}\right), \pi\left(t_{2}\right)\right)$ are profinite over $T$ and $T$ is integral and normal, we have that

$$
\begin{aligned}
\underline{\operatorname{Isom}}_{X}\left(t_{1}, t_{2}\right)(T) & =\underline{\operatorname{Isom}}_{X}\left(t_{1}, t_{2}\right)(k(T)), \\
\underline{\operatorname{Isom}}_{\Pi_{X / k}}\left(\pi\left(t_{1}\right), \pi\left(t_{2}\right)\right)(T) & =\underline{\operatorname{Isom}}_{\Pi_{X / k}}\left(\pi\left(t_{1}\right), \pi\left(t_{2}\right)\right)(k(T)),
\end{aligned}
$$

and hence

since by hypothesis

$$
\underline{\operatorname{Isom}}_{X}\left(t_{1}, t_{2}\right)(T) \stackrel{\sim}{\rightarrow} \underline{\operatorname{Isom}}_{\Pi_{X / k}}\left(\pi\left(t_{1}\right), \pi\left(t_{2}\right)\right)(T)
$$

$$
\underline{\operatorname{Isom}}_{X}\left(t_{1}, t_{2}\right)(k(T)) \stackrel{\sim}{\rightarrow} \underline{\operatorname{Isom}}_{\Pi_{X / k}}\left(\pi\left(t_{1}\right), \pi\left(t_{2}\right)\right)(k(T)) .
$$

Essential surjectivity. Fix a morphism $\tau: T \rightarrow \Pi_{X / k}$. Consider the "universal cover" $\tilde{X}=$ $X \times_{\Pi_{X / k}} T \rightarrow X \times T$, which is an algebraic space thanks to Proposition 5.1: our hypothesis that $X$ is printable implies that for every finitely generated extension $k^{\prime} / k$, the map $\widetilde{X}\left(k^{\prime}\right) \rightarrow T\left(k^{\prime}\right)$ is bijective.

Let $R$ be a DVR over $k$ with fraction and residue fields finitely generated over $k$, and suppose that we have a morphism $\operatorname{Spec} R \rightarrow T$. Since $k(R)$ is finitely generated over $k$, the morphism Spec $k(R) \rightarrow T$ lifts uniquely to $\tilde{X}$. The morphism $\widetilde{X} \rightarrow T$ is separated and universally closed since $\widetilde{X} \rightarrow X \times T$ is profinite (it can be obtained by base change from the diagonal of $\Pi_{X / k}$ ) and $X \times T \rightarrow T$ is proper. Thanks to [Sta20, Lemmas 0A3X, 0A3W and 03KU] this is enough to apply the valuative criterion; that is, we get that $\operatorname{Spec} R \rightarrow T$ lifts uniquely to $\widetilde{X}$. We thus obtain that $\widetilde{X}(R) \rightarrow T(R)$ is bijective too. If $A$ is either a field finitely generated over $k$ or a DVR with fraction and residue fields finitely generated over $k$, denote the inverse map by

$$
\iota: T(A) \rightarrow \widetilde{X}(A) .
$$

Thanks to Proposition 5.1, there exist a finite étale gerbe $\Phi$ and a representable morphism $X \rightarrow \Phi$; the fibre product $X \times_{\Phi} T$ is an algebraic space. The natural morphism $\Pi_{X / k} \rightarrow \Phi$ induces a morphism $\omega: \widetilde{X} \rightarrow X \times_{\Phi} T$. The reader may keep in mind the particular case in which $X$ is an algebraic space and $\Phi=$ Spec $k$ : we use the morphism $X \rightarrow \Phi$ only to "kill" the inertia of $X \times T$.

Now, consider the generic point $\xi: \operatorname{Spec} k(T) \rightarrow T$, and let $S \subseteq X \times_{\Phi} T$ be the closure of $\omega(\iota(\xi)): \operatorname{Spec} k(T) \rightarrow X \times_{\Phi} T$ with the reduced structure and $p: S \rightarrow T$ the projection. We have that $S$ is an integral algebraic space. The situation is illustrated in the following diagram:

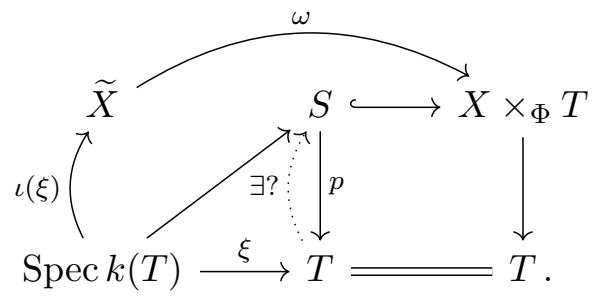

Observe that it is sufficient to prove that $p: S \rightarrow T$ is an isomorphism. In fact, if $S \rightarrow T$ is an isomorphism, we have an induced morphism $x: T \rightarrow X \times_{\Phi} T \rightarrow X$ such that $\pi(x): T \rightarrow \Pi_{X / k}$ is generically isomorphic to the original morphism $\tau: T \rightarrow \Pi_{X / k}$. Thanks to what we have shown in the preceding point, the fact that $\tau$ and $\pi(x)$ are generically isomorphic implies that they are 


\section{GrothendiecK's AnABELIAN CONJECTURES}

isomorphic; hence, $\tau$ is in the essential image of $X(T) \rightarrow \Pi_{X / k}(T)$. Let us show that $p: S \rightarrow T$ is an isomorphism.

Step 1: the map $S\left(k^{\prime}\right) \rightarrow T\left(k^{\prime}\right)$ is injective for any field extension $k^{\prime} / k$. Since $S$ is of finite type over $k$ and all the points of $T$ have residue field finitely generated over $k$, it is enough to do this step for $k^{\prime} / k$ finitely generated. For every point $s$ : Spec $k^{\prime} \rightarrow S$ with $k^{\prime} / k$ finitely generated, consider $\omega(\iota(p(s)))$ : Spec $k^{\prime} \rightarrow X \times_{\Phi} T$. It is enough to show that

$$
\omega(\iota(p(s)))=s,
$$

and it is enough to do so for $k^{\prime}=k(s)$. We prove this by induction on the Krull height of $s$ in $S$ (the height can be defined by passing to an étale neighbourhood which is a scheme).

If $s$ has height 0 , then $s$ is the generic point $\omega(\iota(\xi))$ of $S$ and $p(s)$ maps to the generic point $\xi$ of $T$; thus, $k(T)=k(s)$ and $s=\omega(\iota(\xi))=\omega(\iota(p(s)))$.

If $\operatorname{ht}_{S}(s)>0$, there exists a germ of a non-constant curve on $S$ passing through $s$. More precisely, there exist a noetherian DVR $R$ with fraction and residue fields finitely generated over $k$ and a morphism $r$ : Spec $R \rightarrow S$ such that the closed point maps to $s$ and the open point maps to a point $s_{0} \neq s$. In order to find $R$, take an étale neighbourhood $\left(S^{\prime}, s^{\prime}\right)$ of $s$ which is a scheme, and choose $R$ as the normalization of a dimension 1 integral quotient of $\mathcal{O}_{S^{\prime}, s^{\prime}}$.

Now consider $\omega(\iota(r))$ : Spec $R \rightarrow X \times_{\Phi} T$. We have $\omega(\iota(p(r)))_{k(R)}=r_{k(R)}$ by the induction hypothesis; this implies that $\omega(\iota(p(r)))=r$ since $S \rightarrow T$ is separated. Let $R / \mathfrak{m}$ be the residue field of $R$. We have $k(s) \subseteq R / \mathfrak{m}$ and

$$
\omega(\iota(p(s)))_{R / \mathfrak{m}}=\omega(\iota(p(r)))_{R / \mathfrak{m}}=r_{R / \mathfrak{m}}=s_{R / \mathfrak{m}}
$$

and thus $\omega(\iota(p(s)))=s$. This concludes step 1 .

Step 2: the map $S\left(k^{\prime}\right) \rightarrow T\left(k^{\prime}\right)$ is bijective. We already know that it is injective. Moreover, $T$ is integral and $S \rightarrow T$ is proper; thus, $S \rightarrow T$ is bijective set-theoretically. Let $t \in T$ be any point; we thus know that $S_{t}$ has exactly one point $s \in S_{t}$. Since $S_{t}$ is of finite type over $k(t)$, it follows that $k(s)$ is finite over $k(t)$. We are in characteristic 0 , and thus $k(s)$ is separable over $k(t)$. Since $S_{t}\left(k^{\prime}\right) \rightarrow \operatorname{Spec} k(t)\left(k^{\prime}\right)$ is injective for every extension $k^{\prime} / k$, we get that $k(s)=k(t)$, and this concludes step 2 .

Step 3: $p: S \rightarrow T$ is an isomorphism. Thanks to the previous steps, $S$ is quasi-finite over $T$, and thus it is a scheme; see [Sta20, Proposition 03XX].

If $T$ is quasi-compact and quasi-separated, we can apply Zariski's main theorem [Sta20, Lemma 05K0], and there exists a factorization $S \rightarrow T^{\prime} \rightarrow T$ with $S \rightarrow T^{\prime}$ an open immersion and $T^{\prime} \rightarrow T$ finite. Since $S$ is integral, we may assume $T^{\prime}$ integral too and $k(S)=k\left(T^{\prime}\right)=k(T)$. Since $T$ is normal, $T^{\prime} \rightarrow T$ is an isomorphism. It follows that $S \rightarrow T$ is a bijective open immersion, that is, an isomorphism.

If $T$ is not quasi-compact and quasi-separated, cover it by open affine schemes $T_{i}$ with restrictions $S_{i} \rightarrow T_{i}$. For each $i$, the argument above works since $T_{i}$ is quasi-compact and quasiseparated; hence, $S_{i} \rightarrow T_{i}$ is an isomorphism. It follows that $S \rightarrow T$ is an isomorphism.

Remark 9.2. We cannot hope to remove completely the normality hypothesis from Theorem 9.1. Consider an integral, projective curve $X$ of geometric genus at least 2 with only a cuspidal singularity and smooth normalization $\bar{X}$ over a number field $k$. Passing to $\mathbb{C}$, we may check that $\Pi_{X / k}=\Pi_{\bar{X} / k}$. If the section conjecture holds, $\bar{X}$ is printable: Theorem 9.1 without the normality hypothesis would give us a section $X \rightarrow \bar{X}$. 


\section{G. BRESCIANI}

Corollary 9.3. If smooth, proper, hyperbolic curves satisfy the section conjecture, then they satisfy the hom conjecture.

Proof. If hyperbolic curves satisfy the section conjecture, then they are printable thanks to Proposition 3.7. Hence, they satisfy the hom conjecture thanks to Theorem 9.1.

Thanks to Corollary 9.3, we can also see the anabelian conjecture proved by Mochizuki as a particular case of the section conjecture, rather than a different conjecture.

Theorem 9.1 allows us to prove that the topological fundamental group of a printable DM stack has no abelian finite-index subgroup. We know no other result of the form "if a variety shows anabelian behaviour, then its fundamental group is far from being abelian": conjectures and theorems are always in the other direction.

Proposition 9.4. Let $X$ be a printable DM stack of positive dimension. Then $\pi_{1}\left(X_{\bar{k}}\right)$ has no finite-index abelian subgroups.

Proof. Thanks to Propositions 5.1 and 3.8, up to taking a finite extension of $k$ and a finite étale covering of $X$, we may assume that $X$ is an algebraic space.

Assume for a contradiction that a finite-index abelian subgroup exists. Up to taking another a finite extension of $k$ and finite étale covering of $X$, we may assume that $\pi_{1}\left(X_{\bar{k}}\right)$ is abelian and $X$ has a rational point $x_{0} \in X(k)$. Let $\mathrm{Sm}_{\mathrm{k}}$ be the category of smooth varieties over $k$. Since $X$ is printable, thanks to Theorem 9.1 , the stacks $X$ and $\Pi_{X / k}$ define two naturally equivalent functors $\mathrm{Sm}_{\mathrm{k}}^{\text {op }} \rightarrow$ Set (by taking equivalence classes of $\Pi_{X / k}(T)$ for every $T \in \mathrm{Sm}_{\mathrm{k}}$ ). The fact that the fundamental group of $X_{\bar{k}}$ is abelian implies that the gerbe $\Pi_{X / k}$ is abelian, and hence its functor is enriched in groups with identity $\pi\left(x_{0}\right) \in \Pi_{X / k}\left(x_{0}\right)$; thus, the same is true for the functor defined by $X$ and $x_{0}$.

Now take an étale cover $U \rightarrow X$ with $U$ a scheme, and let $R=U \times_{X} U$. Since $U$ and $R$ are smooth varieties, $X(U)$ and $X(R)$ are groups with the structure inherited from $\Pi_{X / k}(U)$ and $\Pi_{X / k}(R)$. This allows us to construct the usual maps $m: X \times X \rightarrow X$ and $i: X \rightarrow X$ giving a group structure to $X$. Hence, the functor of points of $X$ is enriched in groups over the whole category of schemes over $k$ and not just the smooth ones. This implies that $X$ is not only an algebraic space but also a scheme; see [Art69, Theorem 4.1].

Hence, $X$ is actually a proper group scheme, that is, an abelian variety. But it is well known that an abelian variety of positive dimension is not printable; see, for instance, [Mat12], where a proof is given for elliptic curves (the proof actually works without modifications for positivedimensional abelian varieties).

\section{Elementary anabelian stacks}

Recall that Grothendieck defined a geometrically connected variety $X$ as elementary anabelian if there exists a chain of morphisms

$$
X=X_{N} \rightarrow X_{N-1} \rightarrow \cdots \rightarrow X_{1} \rightarrow X_{0}=\operatorname{Spec} k
$$

such that $X_{i+1} \rightarrow X_{i}$ is a smooth fibration whose fibres are hyperbolic curves; see [Gro97]. We want to extend this definition to elementary anabelian stacks.

Definition 10.1. Let $X$ and $Y$ be DM stacks. A morphism $Y \rightarrow X$ is a family of orbicurves if it is smooth, proper, and its fibres are geometrically connected orbicurves. 


\section{GrothendieCK's ANABELIAN CONJECTURES}

DEFINITION 10.2. Let $k$ be a field of characteristic 0. A DM stack is constructible by fibrations over $k$ if it can be constructed by recursion in the following way:

(i) The scheme Spec $k$ is constructible by fibrations.

(ii) If $Y \rightarrow X$ is a family of hyperbolic orbicurves and $X$ is constructible by fibrations, then $Y$ is constructible by fibrations.

(iii) If $Y \rightarrow X$ is finite, representable and étale, then $X$ is constructible by fibrations if and only if $Y$ is constructible by fibrations.

We say that $X$ is an elementary anabelian stack if there exists a field extension $k^{\prime} / k$ such that $X_{k^{\prime}}$ is constructible by fibrations.

Lemma 10.3. Let $X$ be a DM stack over a field $k$ of characteristic 0 and $K / k$ any extension. Then $X$ is elementary anabelian if and only if $X_{K}$ is elementary anabelian.

Proof. If $X$ is elementary anabelian, there exists an extension $k^{\prime} / k$ such that $X_{k^{\prime}}$ is constructible by fibrations. It is possible to find an extension $K^{\prime} / k$ containing both $k^{\prime}$ and $K$ as subextensions. It follows that $X_{K^{\prime}}$ is constructible by fibrations, and thus $X_{K}$ is elementary anabelian. The other implication is trivial.

Lemma 10.4. Let $X$ be an elementary anabelian stack over a field $k$ of characteristic 0 . There exists a diagram of field extensions

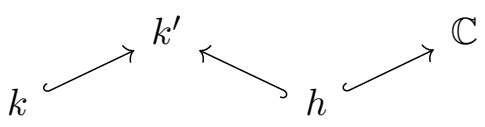

such that the extensions $k^{\prime} / k$ and $h / \mathbb{Q}$ are finitely generated and $X_{k^{\prime}}$ descends to a DM stack $Y$ over $h$ which is constructible by fibrations.

If $k$ is finitely generated over $\mathbb{Q}$ and $k \subseteq \mathbb{C}$, we may choose $k^{\prime}=h$; that is, there exists a finitely generated subextension $\mathbb{C} / k^{\prime} / k$ such that $X_{k^{\prime}}$ is constructible by fibrations.

Proof. The first part follows from standard arguments about finite presentation. For the second part, find $k^{\prime}$ as before and embed it in $\mathbb{C}$ as an extension of $k$ using the fact that $\mathbb{C}$ is algebraically closed of infinite transcendence degree over $k$.

Corollary 10.5. A DM stack over $\mathbb{C}$ is an elementary anabelian stack if and only if it is constructible by fibrations.

Proof. The "if" part is by definition. Let $X$ be elementary anabelian over $\mathbb{C}$. It descends to a DM stack $Y$ over some finitely generated subfield $k \subseteq \mathbb{C}$. Apply Lemma 10.4 to $Y$; we find a finitely generated subextension $\mathbb{C} / k^{\prime} / k$ such that $Y_{k^{\prime}}$ is constructible by fibrations. It follows that $Y_{\mathbb{C}}=X$ is constructible by fibrations.

\subsection{Topology of elementary anabelian stacks}

It will be useful to define and study the topological counterparts of elementary anabelian stacks. Mainly, we do so because we will need the long exact sequence of a fibration: in the algebraic setting, the standard reference [Fri73] does not cover stacks. As a workaround, we will pass through topology and then get back to the algebraic setting using Serre's good groups. We refer to [Noo05] and [Noo14] for the theory of topological stacks and orbifolds, fibrations and long exact homotopy sequences. 


\section{G. BRESCIANI}

Definition 10.6. A topological orbicurve is a complete orbifold $X$ of dimension 2 with a coarse moduli space $X \rightarrow S$ which restricts to an isomorphism on the complement of a finite subset of $S$ and in which $S$ is a compact, orientable surface.

Topological orbicurves have a rational Euler characteristic analogously to algebraic orbicurves; thus, we may define hyperbolic, elliptic and parabolic topological orbicurves.

DEFINITION 10.7. Elementary anabelian topological stacks are topological DM stacks defined by recursion in the following way:

(i) The point is elementary anabelian.

(ii) If $Y \rightarrow X$ is a fibration whose fibres are hyperbolic topological orbicurves and $X$ is elementary anabelian, then $Y$ is elementary anabelian.

(iii) If $Y \rightarrow X$ is a finite covering space, then $X$ is elementary anabelian if and only if $Y$ is elementary anabelian.

In order to pass from the topological to the algebraic setting, we need to check that all groups involved are good in the sense of Serre. Recall that a discrete group $G$ is good in the sense of Serre if the natural homomorphism

$$
\mathrm{H}^{q}(\widehat{G}, M) \rightarrow \mathrm{H}^{q}(G, M)
$$

is an isomorphism for every finite $G$-module $M$, where $\widehat{G}$ is the profinite completion of $G$. The reason why we are interested in good groups is the following: if $G / K=H$ is an extension with $G$ good and $H$ finitely generated, then

$$
1 \rightarrow \widehat{K} \rightarrow \widehat{G} \rightarrow \widehat{H} \rightarrow 1
$$

is exact; see [Ser94, § I.2.6, Exercises 1 and 2].

Finite groups are obviously good, and fundamental groups of compact, orientable surfaces are known to be good: this was already known to Serre when he introduced the concept; see [SCM04, Letter October 22, 1961, p. 138]. In [GJZ08, Proposition 3.7], it is proved that fundamental groups of topological orbicurves are good.

We now need to strengthen the concept of good groups in order to obtain a definition which is stable under extension. We will do so by requesting that our groups are of type $\mathrm{FL}_{\infty}$. Recall that a group $G$ is of type $\mathrm{FL}_{\infty}$ if $\mathbb{Z}$ as a $\mathbb{Z}[G]$-module has a resolution by free $\mathbb{Z}[G]$-modules of finite rank; see [Bro94, Chapter VIII]. Groups of type $\mathrm{FL}_{\infty}$ are finitely generated; see [Bro94, $\S$ VIII.4, Exercise 1]. If a group $G$ is of type $\mathrm{FL}_{\infty}$ and $M$ is a finite $G$-module, then $\mathrm{H}^{q}(G, M)=$ $\operatorname{Ext}_{\mathbb{Z}[G]}^{q}(\mathbb{Z}, M)$ is finite since it is torsion; we can use the finite rank resolution of $\mathbb{Z}$ to show that it is finitely generated.

Finite groups are of type $\mathrm{FL}_{\infty}$ since the resolution can easily be constructed step by step. Moreover, if there exists a CW complex $X$ of type $K(G, 1)$ whose $n$-skeleton is finite for every $n$, then the cellular chain complex of the universal covering of $X$ shows that $G$ is of type $\mathrm{FL}_{\infty}$; see [Bro94, § I.4]. In particular, the fundamental group of surfaces is of type $\mathrm{FL}_{\infty}$.

DEFINITION 10.8. We say that a group is very good if it is good and of type $\mathrm{FL}_{\infty}$.

Lemma 10.9. Let $H=G / K$ be an extension of groups. If $H$ and $K$ are very good, then $G$ is very good.

Proof. If $H$ and $K$ are of type $\mathrm{FL}_{\infty}$, it is known that $G$ is of type $\mathrm{FL}_{\infty}$; see [Wal61]. 


\section{GrothendiECK'S ANABELIAN CONJECTURES}

If $H$ and $K$ are good, a sufficient condition for $G$ to be good is that $K$ is finitely generated and $\mathrm{H}^{q}(K, M)$ is finite for every finite $G$-module $M$; see [Ser94, $\S$ I.2.6, Exercises 1 and 2]. If $K$ is of type $\mathrm{FL}_{\infty}$, this is automatically satisfied.

Recall that two groups are called commensurable if they have isomorphic finite-index subgroups.

Lemma 10.10. If $H$ and $G$ are commensurable and $G$ is very good, then $H$ is very good.

Proof. The fact that $H$ is good is proved in [GJZ08, Lemma 3.2]. We want to show that it is $\mathrm{FL}_{\infty}$ too. First suppose that $H$ is a finite-index subgroup of $G$. Since $\mathbb{Z}[G]$ is a free $\mathbb{Z}[H]$-module of finite rank, it is immediate to check that $H$ is of type $\mathrm{FL}_{\infty}$. Now suppose that $G$ is a finite-index subgroup of $H$. By the preceding case, we may reduce to the case in which $G$ is normal of finite index in $H$. The statement then follows from Lemma 10.9. The general case directly follows from the first two.

Lemma 10.11. Let $X$ be an elementary anabelian topological stack. Then

(i) $X$ is of type $K(G, 1)$;

(ii) $X$ is uniformizable; that is, there exists a finite covering space $Y \rightarrow X$ with $Y$ a manifold;

(iii) $\pi_{1}(X)$ is very good and residually finite.

Proof. Let us prove this by recursion using the rules defining elementary anabelian topological stacks.

If $X$ is just a point, then it clearly satisfies the assertion.

Let $Y \rightarrow X$ be a finite covering space. Clearly, $Y$ is of type $K(G, 1)$ and uniformizable if and only if the same holds for $X$. By Lemma 10.10, the group $\pi_{1}(X)$ is very good if and only if the same holds for $\pi_{1}(Y)$. If $H=G / K$ is a finite group, then it is immediate to show that $G$ is residually finite if and only if $K$ is residually finite. Using a Galois closure $Z \rightarrow Y \rightarrow X$, we get that $\pi_{1}(X)$ is residually finite if and only if the same holds for $\pi_{1}(Y)$.

If $X$ is a complete, orientable surface of genus at least 2 , it is clearly of type $K(G, 1)$ and uniformizable. The fact that $\pi_{1}(X)$ is good is proved in [GJZ08, Proposition 3.7]. Since $X$ is of type $K(G, 1)$ and we can endow it with a structure of finite CW complex, it follows that $\pi_{1}(X)$ is of type $\mathrm{FL}_{\infty}$ and thus very good. The fact that $\pi_{1}(X)$ is residually finite is well known; see $[$ Hem72].

Let $X$ be a hyperbolic topological orbicurve. Thanks to [BN06, Proposition 5.1, Corollary 5.9], there exists some finite covering space $Y \rightarrow X$ with $Y$ a hyperbolic surface; thus, the assertion follows from the preceding case.

Now suppose that $X$ satisfies the assertion, and let $Y \rightarrow X$ be a fibration whose fibres are hyperbolic topological orbicurves, and let $F$ be a fibre. We have a commutative diagram

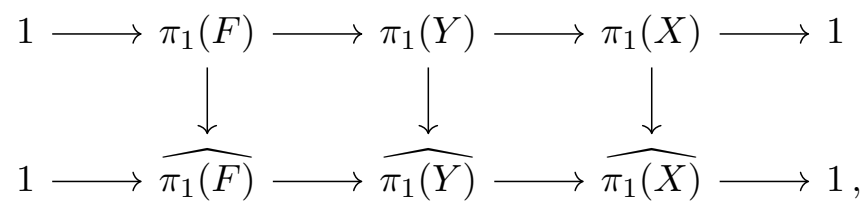

where the first row is exact, being the long exact sequence of the fibration. It follows that $Y$ is of type $K(G, 1)$. Since $\pi_{1}(X)$ and $\pi_{1}(F)$ are very good, $\pi_{1}(Y)$ is very good too thanks to Lemma 10.9, and the second row is exact. Moreover, the left and right vertical arrows are injective 


\section{G. Bresciani}

since $\pi_{1}(F)$ and $\pi_{1}(X)$ are residually finite; thus, the central vertical arrow is injective by diagram chasing, and hence $\pi_{1}(Y)$ is residually finite.

It remains to show that $Y$ is uniformizable. Since $X$ is uniformizable, up to passing to a finite covering space, we may assume that $X$ is a manifold. We know that $F$ is uniformizable. Let $F^{\prime} \rightarrow F$ be a finite covering space which is a surface, and let $Q \subseteq \widehat{\pi_{1}(F)}$ be the set-theoretic

complement of $\widehat{\pi_{1}\left(F^{\prime}\right)}$. Since $\pi_{1}\left(F^{\prime}\right)$ has finite index in $\pi_{1}(F)$, we have that $Q \subseteq \widehat{\pi_{1}(F)}$ is compact. By the injectivity of $\widehat{\pi_{1}(F)} \rightarrow \widehat{\pi_{1}(Y)}$, the image of $Q$ in $\widehat{\pi_{1}(Y)}$ does not contain 1. By the compactness of $Q$, it follows that there exists a finite quotient $\widehat{\pi_{1}(Y)} \rightarrow G$ such that the image of $Q$ in $G$ does not contain 1. Let $Y^{\prime} \rightarrow Y$ be the associated $G$-covering. Let us show that $Y^{\prime}$ is a manifold.

In fact, let $K \subseteq \widehat{\pi_{1}(F)}$ be the kernel of the composition $\widehat{\pi_{1}(F)} \rightarrow \widehat{\pi_{1}(Y)} \rightarrow G$. Since the image of $Q$ in $G$ does not contain 1, we have that $K \subseteq \widehat{\pi_{1}\left(F^{\prime}\right)}$. Hence, the associated (possibly disconnected) $G$-covering $F^{\prime \prime} \rightarrow F$ dominates $F^{\prime}$ and is thus a manifold. In particular, $Y^{\prime} \rightarrow X$ is a fibration whose base and fibres are manifolds, and thus it is a manifold too.

COROllary 10.12. The étale homotopy type of an elementary anabelian stack over an algebraically closed field of characteristic 0 is $K(G, 1)$.

Proof. Thanks to Lemma 10.4, we may reduce to the case in which $k=\mathbb{C}$ and $X$ is constructible by fibrations. Let $X$ be an elementary anabelian stack; the associated topological stack $X^{\text {an }}$ in the sense of [Noo05] is an elementary anabelian topological stack. Now apply [AM69, Theorem 6.7] and Lemma 10.11.

\subsection{An additional property of elementary anabelian stacks}

The class of elementary anabelian stacks is by definition stable under a certain number of operations. There is another natural, non-obvious operation under which they are stable: if $Y \rightarrow X$ is an elementary anabelian stack and $Y \rightarrow X$ is a proper étale morphism, for instance a proper étale gerbe, then $Y$ is elementary anabelian.

We remark that this property is not strictly necessary for the main purposes of the paper: it just seems appropriate to highlight it while introducing elementary anabelian stacks.

We refer to $[$ Sta20, §06QB] for the definition and main properties of gerbes.

Lemma 10.13. Let $Y \rightarrow X$ be a morphism of finite type of algebraic stacks which is a gerbe. The following are equivalent:

(i) The morphism $Y \rightarrow X$ is proper étale.

(ii) The morphism $Y \rightarrow X$ is separated and DM.

(iii) The diagonal $Y \rightarrow Y \times_{X} Y$ is finite étale.

(iv) The relative inertia $I_{Y / X} \rightarrow Y$ is finite étale.

Proof. (i) $\Rightarrow$ (ii) This holds by definition.

(ii) $\Rightarrow$ (iii) Since $Y \rightarrow X$ is separated and DM, the diagonal is proper and unramified. Since the diagonal of a gerbe is always flat [Sta20, Lemma 0CPR], we conclude.

(iii) $\Rightarrow$ (i) Since $Y \rightarrow X$ is gerbe, it is a universal homeomorphism by [Sta20, Lemma 06R9]; it is separated since the diagonal is finite; and it is of finite type by hypothesis. Thus, it is proper. Moreover, $Y \rightarrow X$ is flat since it is a gerbe [Sta20, Proposition 0CPS] and unramified since it is of finite type with étale diagonal [Sta20, Lemma 0CJ0]; thus, we conclude that it is étale too. 


\section{GrothendieCK's ANABELIAN CONJECTURES}

(iii) $\Leftrightarrow$ (iv) Since $Y \rightarrow X$ is gerbe, the diagonal is an fppf covering by [Sta20, Proposition 0CPS]. It follows that the diagonal is finite étale if and only if the same holds for the relative inertia.

If $\Phi \rightarrow$ Spec $k$ is a proper étale gerbe over a field $k$, then it is immediate to check that it is a finite étale gerbe in the sense of [BV15].

Lemma 10.14. Let $f: Y \rightarrow X$ be a proper étale morphism of DM stacks that is locally of finite type with Stein factorization

$$
Y \rightarrow \operatorname{Spec} f_{*} \mathcal{O}_{Y} \rightarrow X
$$

Then $Y \rightarrow \operatorname{Spec} f_{*} \mathcal{O}_{Y}$ is a proper étale gerbe and Spec $f_{*} \mathcal{O}_{Y} \rightarrow X$ is representable finite étale.

Proof. Up to passing to an étale cover and since $f_{*}$ commutes with étale base change, we may assume that $X$ is a scheme. If $X$ is a scheme, the inertia stack $I_{Y}$ of $Y$ coincides with the relative inertia stack $I_{Y / X}$; see [Sta20, Lemma 04Z6]. Since $Y \rightarrow X$ is étale, it follows that $I_{Y} \simeq I_{Y / X}$ is étale over $Y$ thanks to [Sta20, Lemma 0CJ0]. In particular, $I_{Y}$ is flat over $Y$, and thus $Y$ is a gerbe over its coarse moduli sheaf $M$, which is an algebraic space too; see [Sta20, Proposition 06QJ] and [Sta20, Lemma 06QD].

Since $f$ is proper and $X$ is locally of finite type, pushforward of coherent sheaves is coherent, see [Fal03], and hence $\operatorname{Spec} f_{*} \mathcal{O}_{Y} \rightarrow X$ is a finite morphism. We have a natural morphism $M \rightarrow$ Spec $f_{*} \mathcal{O}_{Y}$ since $X$, and thus Spec $f_{*} \mathcal{O}_{Y}$, is a scheme. On the other hand, $M \rightarrow X$ is proper and quasi-finite, hence affine, and this gives us a natural morphism in the other direction, Spec $f_{*} \mathcal{O}_{Y} \rightarrow M$. These morphisms are easily checked to be inverses.

Thus, we know that $Y \rightarrow M=\operatorname{Spec} f_{*} \mathcal{O}_{Y}$ is a gerbe and $M \rightarrow X$ is finite. Since $Y \rightarrow X$ is separated and DM and $M \rightarrow X$ is representable and finite, by [Sta20, Lemma 050M], we get that $Y \rightarrow M$ is separated and DM and thus proper étale by Lemma 10.13. It remains to prove that $M \rightarrow X$ is étale; this follows from the fact that both $Y \rightarrow M$ and $Y \rightarrow X$ are surjective étale.

LEMmA 10.15. If $X$ is an elementary anabelian stack and $f: Y \rightarrow X$ is a proper étale morphism, then $Y$ is an elementary anabelian stack.

Proof. Thanks to Lemma 10.4, we may reduce to the case in which $k=\mathbb{C}$ and $X$ is constructible by fibrations, and we want to show that $Y$ is constructible by fibrations too. Thanks to Lemma 10.14, we may furthermore reduce to the case in which $Y \rightarrow X$ is a proper étale gerbe.

Consider a geometric point $y \in Y(\mathbb{C})$ and its image $x \in X(\mathbb{C})$. The fibre $Y_{x}$ is a gerbe of the form $B G$ for some finite group $G$. Since $Y \rightarrow X$ is a proper étale gerbe, the diagonal $Y \rightarrow Y{ }_{X} Y$ is finite étale. Passing to the associated topological stacks in the sense of [Noo05, Noo14], this tells us that $Y^{\text {an }} \rightarrow X^{\text {an }}$ is a fibration with fibre $B G^{\text {an }}$, and we may thus consider the topological homotopy exact sequence

$$
1 \rightarrow G \rightarrow \pi_{1}^{\mathrm{top}}\left(Y^{\mathrm{an}}\right) \rightarrow \pi_{1}^{\mathrm{top}}\left(X^{\mathrm{an}}\right) \rightarrow 1
$$

where $\pi_{2}^{\text {top }}(X)$ is 0 by Lemma 10.11 . Since $G$ and $\pi_{1}^{\text {top }}(X)$ are very good, we can pass to profinite completions

$$
1 \rightarrow G \rightarrow \widehat{\pi_{1}^{\mathrm{top}}\left(Y^{\mathrm{an}}\right)}=\pi_{1}(Y) \rightarrow \widehat{\pi_{1}^{\mathrm{top}}\left(X^{\mathrm{an}}\right)}=\pi_{1}(X) \rightarrow 1 .
$$

Since $G$ is finite, there exists a connected, finite étale cover $Z \rightarrow Y$ such that $\pi_{1}(Z) \cap G=\{1\} \subseteq$ $\pi_{1}(Y)$. 


\section{G. BRESCIANI}

Now consider the composition $g: Z \rightarrow Y \rightarrow X$; it is a proper étale morphism. Let us show that it is representable. Consider the Stein factorization

$$
Z \rightarrow \operatorname{Spec} g_{*} \mathcal{O}_{Z} \rightarrow X
$$

it is enough to show that $Z \rightarrow \operatorname{Spec} g_{*} \mathcal{O}_{Z}$ is an isomorphism. By Lemma 10.14, we know that $Z \rightarrow \operatorname{Spec} g_{*} \mathcal{O}_{Z}$ is a proper étale gerbe and that $\operatorname{Spec} g_{*} \mathcal{O}_{Z} \rightarrow X$ is a finite étale cover. Take any geometric fibre of $Z \rightarrow \operatorname{Spec} g_{*} \mathcal{O}_{Z}$. It has the form $B H$ for some finite group $H$; we want to show that $H$ is trivial.

By the same argument as above, we get an embedding $H \subseteq \pi_{1}(Z) \subseteq \pi_{1}(Y)$, and by construction $H$ maps to the identity in $\pi_{1}(X)$. Since $\pi_{1}(Z)$ intersects the kernel of $\pi_{1}(Y) \rightarrow \pi_{1}(X)$ trivially, it follows that $H$ is trivial too.

Hence, we have two finite étale covers $Z \rightarrow Y$ and $Z \rightarrow X$; since $X$ is constructible by fibrations, $Z$ and $Y$ are constructible by fibrations too.

\subsection{From curves to elementary anabelian stacks}

ThEOREM 10.16. Elementary anabelian stacks over a field $k$ that is finitely generated over $\mathbb{Q}$ are fff.

If the section conjecture holds for smooth, proper, hyperbolic curves defined over fields that are finitely generated over $\mathbb{Q}$, then elementary anabelian stacks defined over fields that are finitely generated over $\mathbb{Q}$ are printable.

Proof. Thanks to Theorem 6.6 and Lemma 10.4, it is enough to prove the theorem for DM stacks that are constructible by fibrations. We do this only for printability, the argument for fff is analogous. We are going to check that printability is preserved along the elementary operations that define DM stacks constructible by fibrations. We may assume that smooth, proper, hyperbolic orbicurves are printable thanks to Theorem 7.2.

Obviously, Spec $k$ is printable since $\Pi_{\text {Spec } k / k}=\operatorname{Spec} k$. If $Y \rightarrow X$ is finite étale, then by Proposition 3.8, the stack $Y$ is printable if and only if $X$ is printable. We only have to check that printability is preserved along families of hyperbolic orbicurves.

Let $Y \rightarrow X$ be a family of hyperbolic orbicurves, and assume that $X$ is printable. Denote the fibre product $X \times_{\Pi_{X / k}} \Pi_{Y / k}$ by $\Pi_{Y / X}$; we have a natural 2-commutative diagram

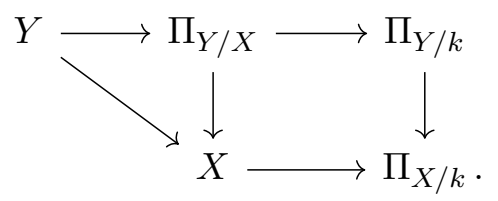

For any extension $k^{\prime} / k$ and morphism $x: \operatorname{Spec} k^{\prime} \rightarrow X$, consider the fibre

$$
\Pi_{Y / X, x}=\Pi_{Y / X} \times_{X} \operatorname{Spec} k^{\prime}=\Pi_{Y / k} \times_{\Pi_{X / k}} \operatorname{Spec} k^{\prime} .
$$

There is a natural map $Y_{x} \rightarrow \Pi_{Y / X, x}$.

Claim: $Y_{x} \rightarrow \Pi_{Y / X, x}$ is the étale fundamental gerbe of $Y_{x}$. Thanks to Proposition A.23, we may assume that $k^{\prime}=k=\bar{k}$ is algebraically closed. Fix a base point $y \in Y_{x}$. Then, since $X$ has trivial topological second homotopy group, there is an exact sequence of étale fundamental groups

$$
0 \rightarrow \pi_{1}^{\mathrm{top}}\left(Y_{x}, y\right) \rightarrow \pi_{1}^{\mathrm{top}}(Y, y) \rightarrow \pi_{1}^{\mathrm{top}}(X, x) \rightarrow 0
$$




\section{GrothendiecK's AnABELIAN CONJECTURES}

Since $\pi_{1}^{\text {top }}(X, x)$ is good in the sense of Serre thanks to Lemma 10.11 , we may pass to profinite completions, that is, étale fundamental groups:

$$
0 \rightarrow \pi_{1}\left(Y_{x}, y\right) \rightarrow \pi_{1}(Y, y) \rightarrow \pi_{1}(X, x) \rightarrow 0 .
$$

Since $\Pi_{Y / X, x}=\Pi_{Y / k} \times_{\Pi_{X / k}} \operatorname{Spec} k(x)$, there is also a short exact sequence

$$
0 \rightarrow \operatorname{Aut}_{\Pi_{Y / X, x}}(y) \rightarrow \operatorname{Aut}_{\Pi_{Y / k}}(y) \rightarrow \operatorname{Aut}_{\Pi_{X / k}}(x) \rightarrow 0,
$$

and there are natural identifications

$$
\pi_{1}\left(Y_{x}, y\right)=\operatorname{Aut}_{\Pi_{Y_{x} / k}}(y), \quad \pi_{1}(Y, y)=\operatorname{Aut}_{\Pi_{Y / k}}(y), \quad \pi_{1}(X, x)=\operatorname{Aut}_{\Pi_{X / k}}(x) .
$$

These fit in a commutative diagram of short exact sequences, identifying $Y_{x} \rightarrow \Pi_{Y / X, x}$ with the étale fundamental gerbe $Y_{x} \rightarrow \Pi_{Y_{x} / k}$.

So we know that $\Pi_{Y / X, x}$ is the étale fundamental gerbe of $Y_{x}$. Since we are assuming that hyperbolic orbicurves are printable, by working fibrewise, we get that $Y \rightarrow \Pi_{Y / X}$ is an equivalence for every finitely generated extension $k^{\prime} / k$. Moreover, we are assuming that $X\left(k^{\prime}\right) \rightarrow \Pi_{X / k}\left(k^{\prime}\right)$ is an equivalence; thus, the same holds for its base change $\Pi_{Y / X}\left(k^{\prime}\right) \rightarrow \Pi_{Y / k}\left(k^{\prime}\right)$. It follows that the composition $Y\left(k^{\prime}\right) \rightarrow \Pi_{Y / k}\left(k^{\prime}\right)$ is an equivalence, as desired.

Finally, let us give a version of Theorem 10.16 for classical elementary anabelian varieties: for them, we can slightly relax the hypotheses since we can work with a fixed base field. Varying the base field is necessary in the passage from curves to orbicurves but not in that from curves to elementary anabelian varieties.

THEOREM 10.17. Let $k$ be a finitely generated extension of $\mathbb{Q}$. If the section conjecture holds for smooth, proper, hyperbolic curves over $k$, then it holds for proper elementary anabelian varieties over $k$. Moreover, elementary anabelian varieties respect the injectivity part of the section conjecture.

Proof. If we assume that the section conjecture holds for an elementary anabelian variety $X / k$ and for all smooth, proper hyperbolic curves over $k$, and $Y \rightarrow X$ is a fibration in hyperbolic curves, then we can repeat the argument contained in the proof of Theorem 10.16 in order to show that the section conjecture holds for $Y / k$. The injectivity part is analogous.

\section{Appendix. Étale fundamental gerbes}

Almost everything in this appendix is already known to the mathematical community; we claim no originality. In particular, most of the ideas and results are already implicit in [BV15] and in the original paper by Deligne [Del89]. Anyway, we could not find a satisfying reference since [BV15] is mostly concerned with the Nori fundamental gerbe rather than the étale one, and hence the theorems regarding the étale fundamental gerbe are not expressed in the right generality. In particular, the authors of [BV15] always work with inflexible fibred categories, while geometrically connected is the right hypothesis. See also [TZ19, §2-4], where part of what is contained in this appendix is done under minor additional hypotheses.

In addition to putting Borne and Vistoli's work for étale fundamental gerbes in the right generality, we give proofs of two technical results, namely of the fact that in characteristic 0 the étale fundamental gerbe behaves well with respect to any field extension (while in [BV15] only algebraic extensions are treated) and of a result concerning the behaviour of the étale fundamental gerbe under finite étale covers. Again, these are not original ideas, but no proof of them was available in the literature. 


\section{G. BRESCIANI}

We want to stress that our effort to state results in maximal generality is not for its own sake: it just happens to work with rather nasty objects that are not even algebraic stacks, like the infinite root stacks of Section 8. Since the theory works for raw fibred categories without any additional hypothesis, we want to give statements in this generality.

If we say that a stack $X$ is finite over a field $k$, we mean that it has groupoid presentation $R \rightrightarrows U$ with $R$ and $U$ finite over $k$; see [BV15, $\S 4]$. In particular, a finite stack over $k$ is not necessarily representable.

\section{A.1 Connected fibred categories}

Definition A.1 ([TZ19, Definition 2.5]). A fibred category $X$ over $k$ is connected if $\mathrm{H}^{0}\left(X, \mathcal{O}_{X}\right)$ has no non-trivial idempotents.

Definition A.2. If $S$ is a scheme and $X$ is a fibred category, we say that a morphism $X \rightarrow S$ is set-surjective if for every point $s \in S$, there exist a field $\Omega$ and a morphism $\operatorname{Spec} \Omega \rightarrow X$ with image $s$ in $S$.

Lemma A.3. A fibred category $X / k$ is not connected if and only there exists a set-surjective morphism $X \rightarrow \operatorname{Spec} k \sqcup \operatorname{Spec} k$.

Proof. If $X \rightarrow$ Spec $k \sqcup$ Spec $k=$ Spec $k \times k$ is set-surjective, the pullback of $1 \times 0$ is a nontrivial idempotent. On the other hand, if $e \in \mathrm{H}^{0}\left(X, \mathcal{O}_{X}\right)$ is a non-trivial idempotent and $S \rightarrow X$ is a morphism, we can define a morphism $S \rightarrow \operatorname{Spec} k \sqcup$ Spec $k$ by sending $S_{e=0}$ to one point and $S_{e=1}$ to the other one. This defines a morphism $X \rightarrow \operatorname{Spec} k \sqcup \operatorname{Spec} k$. Since $e$ is non-trivial, for some schemes $S$ and $S^{\prime}$ with morphisms $S, S^{\prime} \rightarrow X$, we have $S_{e=0} \neq \emptyset$ and $S_{e=1}^{\prime} \neq \emptyset$; that is, $X \rightarrow \operatorname{Spec} k \sqcup \operatorname{Spec} k$ is set-surjective.

Let $X_{1}$ and $X_{2}$ be two fibred categories over $k$. It is possible to define the disjoint union $X_{1} \sqcup X_{2}$ : if $S$ is a scheme, a morphism $S \rightarrow X_{1} \sqcup X_{2}$ is a decomposition of $S=S_{1} \sqcup S_{2}$ with $S_{1}$ and $S_{2}$ open and closed together with a pair of morphisms $s_{i}: S_{i} \rightarrow X_{i}$.

Definition A.4. We define the clopen topology on the category of schemes as the Grothendieck topology for which a cover $\left\{U_{i} \rightarrow U\right\}_{i}$ is a jointly surjective set of morphisms $U_{i} \rightarrow U$ which are both closed and open immersions.

The clopen topology is very coarse; in particular, it is coarser than the Zariski topology.

Lemma A.5. If $X$ is a connected fibred category over $k$ and $X \simeq X_{1} \sqcup X_{2}$, then either $X_{1}$ or $X_{2}$ is empty. If $X$ is a stack in the clopen topology, the converse hold; that is, we can write it as a non-trivial disjoint union if and only if it is disconnected.

Proof. If $X_{1}$ and $X_{2}$ are both non-empty, $1 \times 0$ in $\mathrm{H}^{0}\left(X, \mathcal{O}_{X}\right)=\mathrm{H}^{0}\left(X_{1}, \mathcal{O}_{X_{1}}\right) \times \mathrm{H}^{0}\left(X_{2}, \mathcal{O}_{X_{2}}\right)$ is a non-trivial idempotent.

Now let $e \in \mathrm{H}^{0}\left(X, \mathcal{O}_{X}\right)$ be a non-trivial idempotent. For every scheme $S$, define

$$
\begin{aligned}
& X_{1}(S)=\left\{s \in X(S) \mid s^{*} e=1 \in \mathrm{H}^{0}\left(S, \mathcal{O}_{S}\right)\right\}, \\
& X_{2}(S)=\left\{s \in X(S) \mid s^{*} e=0 \in \mathrm{H}^{0}\left(S, \mathcal{O}_{S}\right)\right\} .
\end{aligned}
$$

We have a natural morphism $X \rightarrow X_{1} \sqcup X_{2}$ sending a morphism $s: S \rightarrow X$ to the pair $s_{1}, s_{2}$, where $s_{1}$ is the restriction of $s$ to $S_{e=1}$ and $s_{2}$ is the restriction of $s$ to $S_{e=0}$. Since $S_{e=1}$ and $S_{e=0}$ are open subsets of $S$ such that $S_{e=0} \sqcup S_{e=1}=S$, if $X$ is a stack in the clopen topology, we get that $X \rightarrow X_{1} \sqcup X_{2}$ is an equivalence. 


\section{GRothendiECK'S ANABELIAN CONJECTURES}

Remark A.6. If $X$ is an algebraic stack, this is equivalent to asking that the underlying topological space $|X|$ (see [Sta20, Tag 04XE]) be connected. On one hand, if $X=X_{1} \sqcup X_{2}$, then $|X|=$ $\left|X_{1}\right| \sqcup\left|X_{2}\right|$. On the other hand, if $|X|=U_{1} \sqcup U_{2}$ is disconnected, the fact that for every scheme $S$, the natural morphism $|S| \rightarrow|X|$ is continuous allows us to define two fibred categories $X_{1}$ and $X_{2}$ such that $\left|X_{i}\right|=U_{i}$ and $X=X_{1} \sqcup X_{2}$.

\section{A.2 Geometrically connected fibred categories}

If $k^{\prime} / k$ is a finite extension of fields, the Weil restriction along $k^{\prime} / k$ is the right adjoint to the functor of base change along Spec $k^{\prime} \rightarrow$ Spec $k$. More concretely, if $X$ is a fibred category over $k$ and $Y$ is a fibred category over $k^{\prime}$, the Weil restriction $\mathrm{R}_{k^{\prime} / k} Y$ is a fibred category over $k$ with an equivalence of categories

$$
\operatorname{Hom}_{k}\left(X, \mathrm{R}_{k^{\prime} / k} Y\right) \simeq \operatorname{Hom}_{k^{\prime}}\left(X_{k^{\prime}}, Y\right)
$$

that is functorial in $X$ and $Y$. We can construct $\mathrm{R}_{k^{\prime} / k} Y$ as the fibred product Aff $/ k \times_{\text {Aff } / k^{\prime}} Y$. When $Y$ is represented by a quasi-projective scheme, $\mathrm{R}_{k^{\prime} / k} Y$ is represented by a scheme too. If $Y$ is represented by a finite stack and $k^{\prime} / k$ is separable, then $\mathrm{R}_{k^{\prime} / k} Y$ is represented by a finite stack too; see [BV15, Lemma 6.2].

Lemma A.7. Let $k^{\prime} / k$ be a finite, separable extension and $Y$ a finite étale stack over $k^{\prime}$. Then $\mathrm{R}_{k^{\prime} / k} Y$ is a finite étale stack over $k$ too.

Proof. In the proof of [BV15, Lemma 6.2], from a finite groupoid presentation $R \rightrightarrows U$ of $Y$ the authors construct a finite groupoid presentation $R^{\prime} \rightrightarrows U^{\prime}$ of $\mathrm{R}_{k^{\prime} / k} Y$. Following their construction, it is immediate to check that if $R \rightrightarrows U$ is étale, $R^{\prime} \rightrightarrows U^{\prime}$ is étale too.

Recall that a fibred category is concentrated if there exist an affine scheme $U$ and a representable, quasi-separated, quasi-compact and faithfully flat morphism $U \rightarrow X$.

If $X$ is concentrated and $u: U \rightarrow X$ is as above, set $R=U \times_{X} U$; we obtain an fpqc groupoid $\left(r_{1}, r_{2}\right): R \rightrightarrows U$ in algebraic spaces. From standard arguments in descent theory, we get an exact sequence

$$
0 \rightarrow \mathrm{H}^{0}\left(X, \mathcal{O}_{X}\right) \stackrel{u^{*}}{\longrightarrow} \mathrm{H}^{0}\left(U, \mathcal{O}_{U}\right) \stackrel{r_{1}^{*}-r_{2}^{*}}{\longrightarrow} \mathrm{H}^{0}\left(R, \mathcal{O}_{R}\right)
$$

and hence it follows easily that for any field extension $k^{\prime} / k$,

$$
\mathrm{H}^{0}\left(X_{k^{\prime}}, \mathcal{O}_{X_{k^{\prime}}}\right)=\mathrm{H}^{0}\left(X, \mathcal{O}_{X}\right) \otimes_{k} k^{\prime} .
$$

Lemma A.8. Let $X$ be a category fibred over $k$, and $k_{s} / k$ a separable closure. Consider the following:

(i) The category $X_{k^{\prime}} / k^{\prime}$ is connected for every extension $k^{\prime} / k$.

(ii) The category $X_{k_{s}} / k_{s}$ is connected.

(iii) The category $X_{k^{\prime}} / k^{\prime}$ is connected for every finite, separable extension $k^{\prime} / k$.

(iv) The field $k$ is the only étale subalgebra of $\mathrm{H}^{0}\left(X, \mathcal{O}_{X}\right)$.

(v) The scheme $\operatorname{Spec~}^{0}\left(X, \mathcal{O}_{X}\right)$ is geometrically connected.

In general, we have implications (i) $\Leftrightarrow$ (ii) $\Rightarrow$ (iii) $\Leftrightarrow$ (iv) $\Leftrightarrow(\mathrm{v})$. If $X$ is an algebraic space or it is concentrated, then (iii) $\Rightarrow$ (ii) holds too.

Proof. (i) $\Rightarrow$ (ii) This is obvious. 


\section{G. BRESCIANI}

(ii) $\Rightarrow$ (i) Suppose that $X_{k^{\prime}} \rightarrow$ Spec $k^{\prime} \sqcup$ Spec $k^{\prime}$ is a set-surjective morphism. Up to enlarging $k^{\prime}$, we may suppose $k_{s} \subseteq k^{\prime}$. Let $S$ be a scheme over $k_{s}$ and $S \rightarrow X_{k_{s}}$ a morphism. By [Sta20, Tag 0363 and Tag 0383], the morphism $S_{k^{\prime}} \rightarrow S$ is open and induces a bijection of connected components.

In particular, we can write $S=S_{1} \sqcup S_{2}$, where $S_{i, k^{\prime}} \rightarrow S_{k^{\prime}} \rightarrow$ Spec $k^{\prime} \sqcup$ Spec $k^{\prime}$ maps to the $i$ th point for $i=1,2$. This allows us to define a morphism $S \rightarrow \operatorname{Spec} k_{s} \sqcup$ Spec $k_{s}$ whose base change is $S_{k^{\prime}} \rightarrow$ Spec $k^{\prime} \sqcup$ Spec $k^{\prime}$, and thus a morphism $X_{k_{s}} \rightarrow$ Spec $k_{s} \sqcup$ Spec $k_{s}$ whose base change is $X_{k^{\prime}} \rightarrow \operatorname{Spec} k^{\prime} \sqcup \operatorname{Spec} k^{\prime}$. The morphism $X_{k_{s}} \rightarrow \operatorname{Spec} k_{s} \sqcup \operatorname{Spec} k_{s}$ is then clearly set-surjective, which is absurd.

(ii) $\Rightarrow$ (iii) If $X_{k^{\prime}} \rightarrow$ Spec $k^{\prime} \sqcup$ Spec $k^{\prime}$ is set-surjective, then $X_{k_{s}} \rightarrow \operatorname{Spec} k_{s} \sqcup$ Spec $k_{s}$ is set-surjective too.

(iii) $\Rightarrow$ (iv) Suppose that $A \subseteq \mathrm{H}^{0}\left(X, \mathcal{O}_{X}\right)$ is a non-trivial finite étale subalgebra of degree $d>1$ and that there exists a scheme $S$ with a morphism $S \rightarrow X$ such that the composition $S \rightarrow X \rightarrow \operatorname{Spec} A$ is dominant. Now choose a finite separable extension $k^{\prime} / k$ which splits $A$. The base change

$$
X_{k^{\prime}} \rightarrow \operatorname{Spec} A_{k^{\prime}}=\operatorname{Spec} k^{\prime d}
$$

is set-surjective because $S_{k^{\prime}} \rightarrow X_{k^{\prime}} \rightarrow$ Spec $k^{\prime d}$ is set-surjective. But this is absurd since $d>1$ and $X_{k^{\prime}}$ is connected.

(iv) $\Rightarrow$ (iii) Suppose that $k^{\prime} / k$ is a finite separable extension and that we have a set-surjective morphism $X_{k^{\prime}} \rightarrow$ Spec $k^{\prime} \sqcup \operatorname{Spec} k^{\prime}$; this induces a morphism $X \rightarrow \mathrm{R}_{k^{\prime} / k}\left(\operatorname{Spec} k^{\prime} \sqcup \operatorname{Spec} k^{\prime}\right)$. Since $\mathrm{R}_{k^{\prime} / k}\left(\operatorname{Spec} k^{\prime} \sqcup \operatorname{Spec} k^{\prime}\right)$ is a finite étale scheme, by hypothesis we have a factorization

$$
X \rightarrow \operatorname{Spec} k \rightarrow \mathrm{R}_{k^{\prime} / k}\left(\operatorname{Spec} k^{\prime} \sqcup \operatorname{Spec} k^{\prime}\right) \text {. }
$$

But this gives a factorization $X_{k^{\prime}} \rightarrow \operatorname{Spec} k^{\prime} \rightarrow \operatorname{Spec} k^{\prime} \sqcup \operatorname{Spec} k^{\prime}$, which is absurd.

(iv) $\Leftrightarrow(\mathrm{v})$ This is well known.

(iii) $\Rightarrow$ (ii) If $X$ is concentrated, we have

$$
\mathrm{H}^{0}\left(X_{k^{\prime}}, \mathcal{O}_{X_{k^{\prime}}}\right)=\mathrm{H}^{0}\left(X, \mathcal{O}_{X}\right) \otimes_{k} k^{\prime}
$$

for every extension $k^{\prime} / k$; hence, we can reduce to affine schemes for which the result is well known. If $X$ is an algebraic space, this is [Sta20, Lemma 0A17].

Definition A.9. Let $X$ be a fibred category. We say that $X$ is geometrically connected if the equivalent conditions (iii), (iv) and (v) of Lemma A.8 hold for $X$.

\section{A.3 Existence and base change}

Lemma A.10. A stack over $k$ is finite étale if and only if it is étale and of finite type over $k$.

Proof. A finite stack in the sense of [BV15, Section 4] is clearly proper. On the other hand, let $X$ be an étale stack of finite type over $k$. Étale morphisms are by definition DM; thus, $X$ is a DM stack. Let $U \rightarrow X$ be an étale cover of finite type with $U$ a scheme and $R=U \times{ }_{R} U$. By composition, we get that $U$ and $R$ are étale of finite type over $k$. It follows that $X$ is finite étale.

Definition A.11. An fpqc stack $\Gamma$ over a field $k$ is profinite étale if it is the limit of a projective system of finite, étale stacks over $k$, in the sense of [BV15, Definition 3.5].

Remark A.12. In [BV15, Definition 3.5], the authors define the limit of a projective system $\left(\Gamma_{i}\right)_{i}$ of affine fpqc gerbes as a category fibred in groupoids which turns out to be an fpqc stack. 


\section{GRothendiECK'S ANABELIAN CONJECTURES}

Actually, it is straightforward to check that the definition works without any modification for a projective system $\left(\Gamma_{i}\right)$ of categories fibred in groupoids, and if $\Gamma_{i}$ is an fpqc stack for every $i$, then the limit is also an fpqc stack. Moreover, if $\Gamma_{i}$ is an affine fpqc gerbe for every $i$ and the limit is not empty, then the limit is an fpqc gerbe too; see [BV15, Proposition 3.7].

Definition A.13. Let $X$ be a fibred category over $k$ and $\Pi$ a profinite étale gerbe with a morphism $X \rightarrow \Pi$. Then $X \rightarrow \Pi$ is an étale fundamental gerbe if for every finite, étale stack $\Phi$, the functor $\operatorname{Hom}(\Pi, \Phi) \rightarrow \operatorname{Hom}(X, \Phi)$ is an equivalence of categories.

LEMma A.14. Let $X$ be a fibred category with an étale fundamental gerbe $X \rightarrow \Pi$ and $\Phi$ a profinite étale stack. Then $\operatorname{Hom}(\Pi, \Phi) \rightarrow \operatorname{Hom}(X, \Phi)$ is an equivalence of categories. In particular, the étale fundamental gerbe is unique up to a canonical equivalence.

Proof. This is a straightforward application of the definitions of the étale fundamental gerbe and of profinite étale stacks.

The following simple lemma is rather enlightening in the sense that it draws the line between the étale setting and the Nori setting: its failure for finite stacks is what makes Nori's fundamental gerbe subtler than the étale one.

Lemma A.15. Let $\Phi$ be a finite étale stack. Then the natural morphism $\Phi \rightarrow \operatorname{Spec}^{0}\left(\Phi, \mathcal{O}_{\Phi}\right)$ is a gerbe.

Proof. We give an elementary proof. See also [TZ19, Proposition 3.2] for a more technical proof for finite, reduced stacks.

If $k^{s} / k$ is the separable closure, it is easy to check that $\Phi \rightarrow \operatorname{Spec~}^{0}\left(\Phi, \mathcal{O}_{\Phi}\right)$ is a gerbe if and only if $\Phi_{k^{s}} \rightarrow \operatorname{Spec~} \mathrm{H}^{0}\left(\Phi_{k^{s}}, \mathcal{O}_{\Phi_{k^{s}}}\right)$ is a gerbe. Hence, we may suppose that $k$ is separably closed.

Now choose a finite étale groupoid $R \rightrightarrows U$ giving a presentation of $\Phi$. Since $k$ is separably closed and $R$ and $U$ are finite étale, they are simply finite disjoint unions of points. Hence, we can write $\Phi=\sqcup_{i} B G_{i}$, where $G_{i}$ are finite discrete groups. Now it is obvious that $\Phi=\sqcup_{i} B G_{i} \rightarrow$ $\sqcup_{i} \operatorname{Spec} k$ is a gerbe.

Corollary A.16. Let $X$ be a fibred category. Then $X$ is geometrically connected if and only if every morphism $X \rightarrow \Gamma$, where $\Gamma$ is a finite étale stack, has a factorization $X \rightarrow \Gamma^{\prime} \rightarrow \Gamma$, where $\Gamma^{\prime}$ is a finite étale gerbe.

Proof. Suppose that $X$ is geometrically connected. Consider the composition

$$
X \rightarrow \Gamma \rightarrow \operatorname{Spec~}^{0}\left(\Gamma, \mathcal{O}_{\Gamma}\right) .
$$

Since $X$ is geometrically connected and $\mathrm{H}^{0}\left(\Gamma, \mathcal{O}_{\Gamma}\right)$ is finite étale, we have a factorization

$$
X \rightarrow \operatorname{Spec} k \rightarrow \operatorname{Spec~}^{0}\left(\Gamma, \mathcal{O}_{\Gamma}\right) .
$$

Set $\Gamma^{\prime}=\operatorname{Spec} k \times_{\operatorname{Spec} H^{0}\left(\Gamma, \mathcal{O}_{\Gamma}\right)} \Gamma$, we have a factorization $X \rightarrow \Gamma^{\prime} \rightarrow \Gamma$, and $\Gamma^{\prime}$ is a gerbe over Spec $k$ thanks to Lemma A.15.

On the other hand, if $A \subseteq \mathrm{H}^{0}\left(X, \mathcal{O}_{X}\right)$ is a non-trivial étale subalgebra, the natural morphism $X \rightarrow \operatorname{Spec} A$ cannot factor through any finite gerbe.

The following three results are straightforward modifications of results of Borne and Vistoli.

Theorem A.17 ([BV15, Theorem 5.7]). Let $X$ be a fibred category over $k$. Then $X$ has an étale fundamental gerbe if and only if it is geometrically connected. 


\section{G. BRESCIANI}

Proof. Thanks to Corollary A.16, we can replace inflexible fibred categories with geometrically connected ones. See also [TZ19, Proposition 4.3] for a proof under some minor additional hypotheses.

Proposition A.18 ([BV15, Proposition 6.1]). Let $k^{\prime} / k$ be an algebraic and separable extension and $X$ a geometrically connected fibred category over $k$. Suppose that either

(a) $k^{\prime}$ is finite over $k$, or

(b) $X$ is concentrated.

Then $X_{k^{\prime}}$ is geometrically connected over $k^{\prime}$ and $\Pi_{X_{k^{\prime}} / k^{\prime}}=\operatorname{Spec} k^{\prime} \times \Pi_{X / k}$.

Proof. Replace [BV15, Lemma 6.2] with Lemma A.7.

Given two extensions $G$ and $H$ of a group $\Gamma$, we have defined Hom-ext $\operatorname{Hot}_{\Gamma}(G, H)$ as the set of homomorphisms $G \rightarrow H$ of $\Gamma$-extensions modulo the action of $\operatorname{ker}(H \rightarrow \Gamma)$ by conjugacy. It is more natural to consider the category $\operatorname{Hom}_{-} \operatorname{ext}_{\Gamma}(G, H)$ whose objects are said homomorphisms and whose arrows are given by conjugacy with elements of $\operatorname{ker}(H \rightarrow \Gamma)$. Then $\operatorname{Hom}_{-} \operatorname{ext}_{\Gamma}(G, H)$ is the set of isomorphism classes.

Proposition A.19 ([BV15, Proposition 9.3]). Let $X$ be a quasi-compact, quasi-separated and geometrically connected algebraic stack over $k$ with a geometric point $\bar{x}$ : Spec $\Omega \rightarrow X$ and $T$ any geometrically connected scheme with a geometric point $\bar{t}$ : Spec $\Omega \rightarrow X$. There is a (noncanonical) equivalence of categories

$$
\Pi_{X / k}(T) \rightarrow \text { Hom-ext } \Gamma_{\Gamma_{k}}\left(\pi_{1}(T, \bar{t}), \pi_{1}(X, \bar{x})\right)
$$

that composed with the canonical functor $\operatorname{Hom}_{k}(T, X) \rightarrow \Pi_{X / k}(T)$ gives the natural map

$$
\operatorname{Hom}_{k}(T, X) \rightarrow \text { Hom-ext } \Gamma_{k}\left(\pi_{1}(T, \bar{t}), \pi_{1}(X, \bar{x})\right) .
$$

Proof. Replacing Spec $k$ with $T$ simply does not affect the proof given in [BV15].

Now suppose that we are in characteristic 0. Following Borne and Vistoli, we have shown that the étale fundamental gerbe behaves well under algebraic field extensions: we want to show that, actually, it behaves well with respect to any field extension. The idea is to rephrase the theorem in terms of étale fundamental groups and then use the fact that the étale fundamental group is invariant along extensions of algebraically closed fields; see [GR03, Exposé XIII, Proposition 4.6].

Given a field $k$, let $\operatorname{AffGrp}_{k}$ be the category of affine group schemes over $k$, and let FGrp, PFGrp be the categories of finite and profinite classical groups.

Lemma A.20. The functor FGrp $\rightarrow \mathrm{AffGrp}_{k}$ which sends a finite group to the associated constant group scheme extends to a fully faithful functor PFGrp $\rightarrow \mathrm{AffGrp}_{k}$ which preserves limits.

If $k$ is separably closed, the essential image of the functor PFGrp $\rightarrow \operatorname{AffGrp}_{k}$ consists of the full subcategory of profinite étale group schemes.

Proof. Given a profinite group $G$, define $I_{G}$ as the $k$-algebra of continuous homomorphisms $G \rightarrow k$, where $k$ is endowed with the discrete topology. The $k$-algebra $I_{G}$ can be endowed with a Hopf algebra structure analogously to the finite case. Define the associated profinite constant group scheme

$$
\underline{G}=\operatorname{Spec} I_{G} .
$$

Observe that if we write $G=\lim _{i} G_{i}$ as a limit of finite groups, then the natural map $\lim _{i} I_{G_{i}} \rightarrow$ $I_{G}$ is easily checked to be an isomorphism of Hopf algebras. Using this, it is easy to check that 


\section{GrothendiECK'S ANABELIAN CONJECTURES}

$G \mapsto \underline{G}$ defines a functor PFGrp $\rightarrow \operatorname{Affrp}_{k}$ with the desired properties. Once the functor is defined, the second part of the lemma follows from the analogous statement for finite groups.

LEmma A.21. If $G$ and $H$ are profinite étale groups schemes and $k^{\prime} / k$ is an extension of separably closed fields, then the natural functor

$$
\operatorname{Hom}_{k}\left(B_{k} G, B_{k} H\right) \rightarrow \operatorname{Hom}_{k^{\prime}}\left(B_{k^{\prime}} G, B_{k^{\prime}} H\right)
$$

is an equivalence.

Proof. Both categories have the same description in purely group-theoretic terms; let us explain this. Since $k$ is separably closed, thanks to Lemma A.21 there exist profinite groups $G_{c}$ and $H_{c}$ whose associated group schemes over $k$ are $G$ and $H$. Clearly, $G_{k^{\prime}}$ and $H_{k^{\prime}}$ are associated with $G_{c}$ and $H_{c}$ over $k^{\prime}$.

We now define the category $\operatorname{Hom}_{\text {cont }}\left(B G_{c}, B H_{c}\right)$ of "continuous" functors $B G_{c} \rightarrow B H_{c}$ : its objects are just continuous homomorphisms $G_{c} \rightarrow H_{c}$, and every $h \in H_{c}$ defines an arrow $\varphi \rightarrow h^{-1} \varphi h$ for every continuous homomorphism $\varphi: G_{c} \rightarrow H_{c}$.

We have a natural morphism $\operatorname{Hom}_{\text {cont }}\left(B G_{c}, B H_{c}\right) \rightarrow \operatorname{Hom}\left(B_{k} G, B_{k} H\right)$; since $k$ is separably closed, thanks to Lemma A.21, it is immediate to check that this morphism is an equivalence of categories. The same is true over $k^{\prime}$; thus, the assertion follows by 2-commutativity of the obvious diagram.

For the following Lemma A.22, I would like to thank Marc Hoyois, who suggested the use of noetherian approximation in order to reach full generality; see [Mat18].

LEMma A.22. Let $k^{\prime} / k$ be an extension of algebraically closed fields of characteristic 0 . Consider a concentrated fibred category $X$ over $k$ and a finite étale stack $\Phi$ over $k$. Then the natural functor

$$
\operatorname{Hom}_{k}(X, \Phi) \rightarrow \operatorname{Hom}_{k^{\prime}}\left(X_{k^{\prime}}, \Phi_{k^{\prime}}\right)
$$

is an equivalence of categories.

Proof. Let us prove this firstly under the additional hypothesis that $X$ is a scheme of finite type over $k$. Under this hypothesis, connected components are open; hence, we may suppose that $X$ is connected and $\Phi$ is of the form $B G$ for some finite group $G$. Fix any point $x \in X(k)$. Thanks to [GR03, Exposé XIII, Proposition 4.6], we have that $\pi_{1}(X, x)=\pi_{1}\left(X_{k^{\prime}}, x_{k^{\prime}}\right)$.

We thus have

$$
\begin{aligned}
\operatorname{Hom}_{k}\left(X, B_{k} G\right) & =\operatorname{Hom}_{k}\left(B_{k} \pi_{1}(X, x), B_{k} G\right) \\
& =\operatorname{Hom}_{k^{\prime}}\left(B_{k^{\prime}} \pi_{1}\left(X_{k^{\prime}}, s_{k^{\prime}}\right), B_{k^{\prime}} G\right)=\operatorname{Hom}_{k^{\prime}}\left(X_{k^{\prime}}, B_{k^{\prime}} G\right) .
\end{aligned}
$$

Let us now generalize to $X$ a quasi-compact, quasi-separated scheme. By noetherian approximation [TT90, Theorem C.9], we can write $X$ as an inverse $\operatorname{limit} \lim _{i} X_{i}$, with $X_{i}$ of finite type over $k$. Since $\Phi$ is finite,

$$
\operatorname{Hom}_{k}(X, \Phi)=\underset{i}{\lim } \operatorname{Hom}_{k}\left(X_{i}, \Phi\right)=\underset{i}{\lim } \operatorname{Hom}_{k^{\prime}}\left(X_{i, k^{\prime}}, \Phi_{k^{\prime}}\right)=\operatorname{Hom}_{k^{\prime}}\left(X_{k^{\prime}}, \Phi_{k^{\prime}}\right) .
$$

Finally, if $X$ is a concentrated fibred category, let $U$ be a quasi-compact and quasi-separated scheme with a representable, quasi-separated, quasi-compact and faithfully flat morphism $U \rightarrow$ $X$. Set $R=U \times{ }_{X} U$; then $R$ is again quasi-compact and quasi-separated. Let $\operatorname{Hom}(R \rightrightarrows U, \Phi)$ be the category of morphism $U \rightarrow \Phi$ satisfying the usual cocycle condition on $R$. Descent theory 


\section{G. BRESCIANI}

tells us that $\operatorname{Hom}(R \rightrightarrows U, \Phi)$ is naturally equivalent to $\operatorname{Hom}(X, \Phi)$, even if $X$ is not a stack and hence $X \neq[U / R]$. Since $U$ and $R$ are quasi-compact and quasi-separated, by the preceding case, we conclude that

$$
\operatorname{Hom}_{k^{\prime}}\left(X_{k^{\prime}}, \Phi_{k^{\prime}}\right)=\operatorname{Hom}_{k^{\prime}}\left(R_{k^{\prime}} \rightrightarrows U_{k^{\prime}}, \Phi_{k^{\prime}}\right)=\operatorname{Hom}_{k}(R \rightrightarrows U, \Phi)=\operatorname{Hom}_{k}(X, \Phi) .
$$

Proposition A.23. Let $k$ be a field of characteristic 0. If $X$ is a geometrically connected, concentrated fibred category over $k$, then the natural map $\Pi_{X_{k^{\prime}} / k^{\prime}} \rightarrow \Pi_{X / k} \times_{k} k^{\prime}$ is an isomorphism for every field extension $k^{\prime} / k$.

Proof. Thanks to Proposition A.18, it is immediate to reduce to the case in which $k$ and $k^{\prime}$ are both algebraically closed. We have to show that $\Pi_{X / k} \times_{k} k^{\prime}$ has the universal property of the étale fundamental gerbe of $X_{k^{\prime}}$.

Since $k^{\prime}$ is algebraically closed, every finite étale stack over $k^{\prime}$ has the form $\sqcup_{i} B_{k^{\prime}} G_{i}$ for some finite number of finite groups $G_{i}$. In particular, every finite étale stack over $k^{\prime}$ is isomorphic to $\Phi_{k^{\prime}}$ for some finite étale stack $\Phi$ over $k$; hence, it is enough to show that $\Pi_{X / k} \times_{k} k^{\prime}$ has the universal property with respect to stacks of the form $\Phi_{k^{\prime}}$ with $\Phi$ finite étale over $k$.

Now observe that $\Pi_{X / k}$, being a gerbe over Spec $k$, is concentrated: in fact, any morphism Spec $L \rightarrow \Pi_{X / k}$ with $L$ a field is representable, quasi-compact, quasi-separated and faithfully flat. Hence, both $X$ and $\Pi_{X / k}$ are concentrated, and thanks to Lemma A.22, we have

$$
\operatorname{Hom}_{k^{\prime}}\left(X_{k^{\prime}}, \Phi_{k^{\prime}}\right)=\operatorname{Hom}_{k}(X, \Phi)=\operatorname{Hom}_{k}\left(\Pi_{X / k}, \Phi\right)=\operatorname{Hom}_{k^{\prime}}\left(\Pi_{X / k} \times_{k} k^{\prime}, \Phi_{k^{\prime}}\right) .
$$

\section{A.4 Étale coverings of fibred categories}

Lemma A.24. Let $f: Y \rightarrow X$ be a representable, finite étale morphism of fibred categories. If $X$ is connected, then there exists an integer $d$ such that for every scheme $S$ and every morphism $s: S \rightarrow X$, the étale covering $S \times_{X} Y \rightarrow S$ has constant degree $d$.

Proof. If $S$ is a scheme, $s \in X(S)$ an object and $d \geqslant 0$ an integer, the locus $S_{=d}$ of points $p$ of $S$ such that $Y \times_{X} S \rightarrow S$ has degree $d$ over $p$ is an open and closed subscheme of $S$; set $S_{\neq d}=S \backslash S_{=d}$. This allows us to define a morphism $X \rightarrow \operatorname{Spec} k \sqcup$ Spec $k$ sending $S_{=d}$ to the first point and $S_{\neq d}$ to the second point. If there exist morphisms $S, S^{\prime} \rightarrow X$ such that $S_{=d}$ and $S_{\neq d}^{\prime}$ are both non-empty, then $X$ is not connected, which is absurd.

There exist some $d_{0}$ and a morphism $S \rightarrow X$ such that $S_{=d_{0}} \neq \emptyset$; hence, for every morphism $S^{\prime} \rightarrow X$, we have $S_{=d_{0}}^{\prime}=S^{\prime}$; that is, $Y \rightarrow X$ has constant degree $d_{0}$.

In the following, we need to use quotients by group actions of stacks; see [Rom05, Theorem 4.1] for the general existence result.

Proposition A.25. Let $Y \rightarrow X$ be a representable, finite étale morphism of geometrically connected fibred categories. The following natural 2-commutative diagram is 2-cartesian:

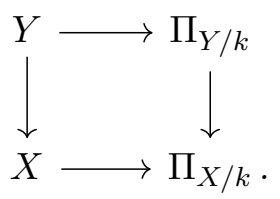

Proof. Thanks to Lemma A.24, the morphism $Y \rightarrow X$ is a finite cover of fixed degree $d$. Let $d \times X$ be the disjoint union of $d$ copies of $X$; we have a finite cover $d \times X \rightarrow X$ of degree $d$. The group $S_{d}$ acts on the fibred category $Z=\underline{\operatorname{Isom}}_{X}(d \times X, Y)$ by automorphisms of $d \times X$, making 


\section{GRothendiECK'S ANABELIAN CONJECTURES}

it into an $S_{d}$-torsor over $X$. If $S$ is a scheme with a morphism $S \rightarrow Z$, we have a trivialization $d \times S \simeq Y \times{ }_{X} S$. The first copy of $d \times S$ gives us a morphism $S \rightarrow Y$, and thus by Yoneda's lemma, we have an $S_{d-1}$-invariant morphism $Z \rightarrow Y$ which is actually an $S_{d-1}$-torsor.

All of this can be summarized by saying that we have a morphism $X \rightarrow B S_{d}$ with identifications $Z=X \times_{B S_{d}}$ Spec $k$ and $Y=X \times_{B S_{d}} B S_{d-1}$. Moreover, define $\Pi=\Pi_{X / k} \times_{B S_{d}} B S_{d-1}$ and $\Lambda=\operatorname{Spec} k \times_{B S_{d}} \Pi_{X / k}$. We have a 2-cartesian diagram

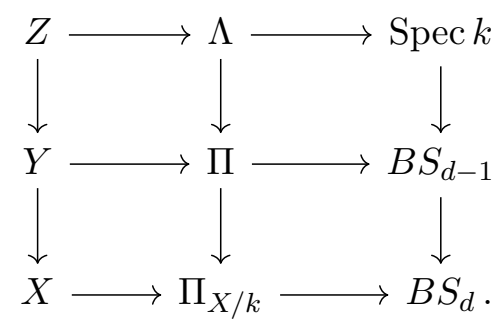

Since $\Pi$ is profinite étale, if we show that it satisfies the universal property of $\Pi_{Y / k}$, then we have that $\Pi=\Pi_{Y / k}$ thanks to Lemma A.14, and hence the assertion.

Now consider a finite étale stack $\Phi$; we want to show that

$$
\operatorname{Hom}_{k}(\Pi, \Phi) \rightarrow \operatorname{Hom}_{k}(Y, \Phi)
$$

is an equivalence of categories. Let $\rho: Z \times S_{d} \rightarrow Z$ be the action. If $Y \rightarrow \Phi$ is a morphism, consider the composition

$$
\rho_{\Phi}: Z \times S_{d} \stackrel{\rho}{\rightarrow} Z \rightarrow Y \rightarrow \Phi .
$$

For every $g \in S_{d}$, this defines a morphism $\rho_{\Phi}(\cdot, g): Z \rightarrow \Phi$. If $h \in S_{d-1} \subseteq S_{d}$, since $Z \rightarrow Y$ is $S_{d-1}$ invariant, we get that $\rho_{\Phi}(\cdot, g)=\rho_{\Phi}(\cdot, g h): Z \rightarrow \Phi$; hence, $\rho_{\Phi}(\cdot,[g])$ is well defined for $[g] \in S_{d} / S_{d-1}$. This gives us an $S_{d}$-equivariant morphism $Z \rightarrow \Phi^{S_{d}} / S_{d-1}$, where $S_{d}$ acts on $\Phi^{S_{d} / S_{d-1}}$ via left multiplication on $S_{d} / S_{d-1}$.

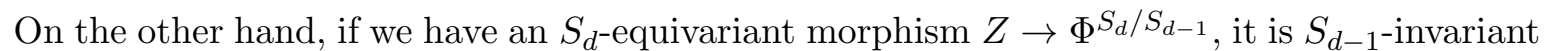
since $S_{d-1}$ acts trivially on $S_{d} / S_{d-1}$. Hence, we have an induced morphism $Y \rightarrow \Phi^{S_{d} / S_{d-1}}$, which, composed with the projection $\Phi^{S_{d} / S_{d-1}} \rightarrow \Phi$ on the identity component, gives a morphism $Y \rightarrow \Phi$. It is easy to check that these constructions are inverses and give an equivalence of categories

$$
\operatorname{Hom}(Y, \Phi) \stackrel{\sim}{\rightarrow} \operatorname{Hom}^{S_{d}}\left(Z, \Phi^{S_{d} / S_{d-1}}\right) .
$$

Since $Z \rightarrow X$ is an $S_{d}$-torsor, we also have an equivalence

$$
\operatorname{Hom}^{S_{d}}\left(Z, \Phi^{S_{d} / S_{d-1}}\right) \stackrel{\sim}{\rightarrow} \operatorname{Hom}_{B S_{d}}\left(X,\left[\Phi^{S_{d} / S_{d-1}} / S_{d}\right]\right)
$$

and the composition

$$
\operatorname{Hom}(Y, \Phi) \stackrel{\sim}{\rightarrow} \operatorname{Hom}_{B S_{d}}\left(X,\left[\Phi^{S_{d} / S_{d-1}} / S_{d}\right]\right) .
$$

We can repeat the same argument with $\Pi_{X / k}, \Pi$ and $\Lambda$ instead of $X, Y$ and $Z$, finding an equivalence

$$
\operatorname{Hom}(\Pi, \Phi) \stackrel{\sim}{\rightarrow} \operatorname{Hom}_{B S_{d}}\left(\Pi_{X / k},\left[\Phi^{S_{d} / S_{d-1}} / S_{d}\right]\right) .
$$

Thanks to Lemma A.10, we have that $\left[\Phi^{\left.S_{d} / S_{d-1} / S_{d}\right]}\right.$ is a finite étale stack, and thus there is another equivalence

$$
\operatorname{Hom}_{B S_{d}}\left(X,\left[\Phi^{S_{d} / S_{d-1}} / S_{d}\right]\right) \stackrel{\sim}{\rightarrow} \operatorname{Hom}_{B S_{d}}\left(\Pi_{X / k},\left[\Phi^{S_{d} / S_{d-1}} / S_{d}\right]\right) .
$$

Composing these three, we obtain the desired equivalence $\operatorname{Hom}(Y, \Phi) \stackrel{\sim}{\rightarrow} \operatorname{Hom}(\Pi, \Phi)$. 


\section{G. BRESCIANI}

\section{ACKNOWLEDGEMENTS}

This article is part of my $\mathrm{PhD}$ thesis. I would like to thank my $\mathrm{PhD}$ adviser Angelo Vistoli for many useful discussions and for teaching me how to use stacks (and almost everything else). I would also like to thank Tamás Szamuely and Hélène Esnault for many useful remarks, and Marc Hoyois for pointing out to me how to use noetherian approximation in order to reach full generality in Lemma A.22; see [Mat18]. Finally, I would like to thank an anonymous referee for carefully reading an earlier draft of the paper and helping me to greatly clarify the exposition.

\section{REFERENCES}

AGV08 D. Abramovich, T. Graber and A. Vistoli, Gromov-Witten theory of Deligne-Mumford stacks, Amer. J. Math. 130 (2008), no. 5, 1337-1398; doi:10.1353/ajm.0.0017.

AM69 M. Artin and B. Mazur, Etale homotopy, Lecture Notes in Math., vol. 100 (Springer-Verlag, Berlin - New York, 1969); doi:10.1007/BFb0080957.

Art69 M. Artin, Algebraization of formal moduli. I, Global Analysis (Papers in Honor of K. Kodaira) (University Tokyo Press, Tokyo, 1969), 21-71.

BE14 N. Borne and M. Emsalem, Un critère d'épointage des sections $\ell$-adiques, Bull. Soc. Math. France 142 (2014), no. 3, 465-487; doi:10.24033/bsmf. 2671.

BN06 K. Behrend and B. Noohi, Uniformization of Deligne-Mumford curves, J. reine angew. Math. 599 (2006), 111-153; doi:10.1515/CRELLE.2006.080.

Bor09 N. Borne, Sur les représentations du groupe fondamental d'une variété privée d'un diviseur à croisements normaux simples, Indiana Univ. Math. J. 58 (2009), no. 1, 137-180; doi:10.1512/ iumj . 2009.58.3734.

Bro94 K. S. Brown, Cohomology of groups, Grad. Texts in Math., vol. 87 (Springer-Verlag, New York, 1994); doi:10.1007/978-1-4684-9327-6.

BV15 N. Borne and A. Vistoli, The Nori fundamental gerbe of a fibered category, J. Algebraic Geom. 24 (2015), no. 2, 311-353; doi:10.1090/S1056-3911-2014-00638-X.

Del89 P. Deligne, Le groupe fondamental de la droite projective moins trois points, Galois Groups over Q (Berkeley, CA, 1987), Math. Sci. Res. Inst. Publ., vol. 16 (Springer, New York, 1989), 79-297; doi:10.1007/978-1-4613-9649-9_3.

EH08 H. Esnault and P. H. Hai, Packets in Grothendieck's section conjecture, Adv. Math. 218 (2008), no. 2, 395-416; doi:10.1016/j.aim.2007.12.009.

Fal03 G. Faltings, Finiteness of coherent cohomology for proper fppf stacks, J. Algebraic Geom. 12 (2003), no. 2, 357-366; doi:10.1090/S1056-3911-02-00321-1.

Fri73 E. M. Friedlander, Fibrations in etale homotopy theory, Publ. Math. Inst. Hautes Études Sci. (1973), no. 42, 5-46; doi:10.1007/BF02685876.

GJZ08 F. Grunewald, A. Jaikin-Zapirain and P. A. Zalesskii, Cohomological goodness and the profinite completion of Bianchi groups, Duke Math. J. 144 (2008), no. 1, 53-72; doi:10.1215/ 00127094-2008-031.

GR03 A. Grothendieck and M. Raynaud, Revêtements étales et groupe fondamental (SGA 1), Doc. Math. (Paris), vol. 3 (Soc. Math. de France, Paris, 2003).

Gro97 A. Grothendieck, Brief an G. Faltings, in Geometric Galois Actions, 1, London Math. Soc. Lecture Note Ser., vol. 242 (Cambridge Univ. Press, Cambridge, 1997), 49-58 (with an English translation on pp. 285-293).

Hem72 J. Hempel, Residual finiteness of surface groups, Proc. Amer. Math. Soc. 32 (1972), 323; doi: $10.2307 / 2038357$. 


\section{GrothendiECK'S ANABELIAN CONJECTURES}

Mat12 MathOverflow, naf (https://mathoverflow.net/users/519/naf), response to Why does the Section Conjecture exclude curves of genus 1?, version: 2012-04-03, https://mathoverflow.net/ q/92992.

Mat18 MathOverflow, M. Hoyois (https://mathoverflow.net/users/20233/marc-hoyois), response to Finite étale covers of concentrated schemes and extension of base field, version: 2018-03-10, https://mathoverflow.net/q/294852.

Moc99 S. Mochizuki, The local pro-p anabelian geometry of curves, Invent. Math. 138 (1999), no. 2, 319-423; doi:10.1007/s002220050381.

Noo04 B. Noohi, Fundamental groups of algebraic stacks, J. Inst. Math. Jussieu 3 (2004), no. 1, 69-103; doi:10.1017/S1474748004000039.

Noo05_, Foundations of topological stacks I, 2005, arXiv:math.AG/0503247.

Noo14 Fibrations of topological stacks, Adv. Math. 252 (2014), 612-640; doi:10.1016/j. aim. 2013.11.008.

Rom05 M. Romagny, Group actions on stacks and applications, Michigan Math. J. 53 (2005), no. 1, 209-236; doi:10.1307/mmj/1114021093.

SCM04 J.-P. Serre, P. Colmez and C. Maclean (eds), Grothendieck-Serre correspondence, bilingual edn (Amer. Math. Soc., Providence, RI, 2004).

Ser94 J.-P. Serre, Cohomologie galoisienne, 5th edn, Lecture Notes in Math., vol. 5 (Springer-Verlag, Berlin, 1994); doi:10.1007/BFb0108758.

Sta20 The Stacks Project Authors, Stacks Project, version 2020, available at https://stacks.math. columbia.edu/.

Sti12 J. Stix, On cuspidal sections of algebraic fundamental groups, in Galois-Teichmüller theory and arithmetic geometry, Adv. Stud. Pure Math., vol. 63 (Math. Soc. Japan, Tokyo, 2012), 519-563; doi:10.2969/aspm/06310519.

Sti13 , Rational points and arithmetic of fundamental groups. Evidence for the section conjecture, Lecture Notes in Math., vol. 2054 (Springer, Heidelberg, 2013); doi:10.1007/ 978-3-642-30674-7.

TT90 R. W. Thomason and T. Trobaugh, Higher algebraic K-theory of schemes and of derived categories in The Grothendieck Festschrift, Vol. III, Progr. Math., vol. 8 (Birkhäuser Boston, Boston, MA, 1990), 247-435; doi:10.1007/978-0-8176-4576-2_10.

TV18 M. Talpo and A. Vistoli, Infinite root stacks and quasi-coherent sheaves on logarithmic schemes, Proc. Lond. Math. Soc. 116 (2018), no. 5, 1187-1243; doi:10.1112/plms.12109.

TZ19 F. Tonini and L. Zhang, Algebraic and Nori fundamental gerbes, J. Inst. Math. Jussieu 18 (2019), no. 4, 855-897; doi:10.1017/s147474801700024x.

Wal61 C.T.C. Wall, Resolutions for extensions of groups, Proc. Cambridge Philos. Soc. 57 (1961), 251-255; doi:10.1017/s0305004100035155.

Giulio Bresciani gbresciani@zedat.fu-berlin.de

Freie Universität Berlin, Arnimallee 3, 14195, Berlin, Germany 